\title{
On wave breaking for Boussinesq-type models
}

\author{
M. Kazolea and M.Ricchiuto \\ Team CARDAMOM, Inria Bordeaux - Sud-Ouest \\ 200 av. de la vieille tour, 33405 Talence cedex, France
}

\begin{abstract}
We consider the issue of wave breaking closure for Boussinesq type models, and attempt at providing some more understanding of the sensitivity of some closure approaches to the numerical set-up, and in particular to mesh size. For relatively classical choices of weakly dispersive propagation models, we compare two closure strategies. The first is the hybrid method consisting in suppressing the dispersive terms in breaking regions, as initially suggested by Tonelli and Petti in 2009. The second is an eddy viscosity approach based on the solution of a a turbulent kinetic energy. The formulation follows early work by O. Nwogu in the 90's, and some more recent developments by Zhang and co-workers (Ocean Mod. 2014), adapting it to be consistent with the wave breaking detection used here. We perform a study of the behavior of the two closures for different mesh sizes, with attention to the possibility of obtaining grid independent results. Based on a classical shallow water theory, we also suggest some monitors to quantify the different contributions to the dissipation mechanism, differentiating those associated to the scheme from those of the partial differential equation. These quantities are used to analyze the dynamics of dissipation in some classical benchmarks, and its dependence on the mesh size. Our main results show that numerical dissipation contributes very little to the the results obtained when using eddy viscosity method. This closure shows little sensitivity to the grid, and may lend itself to the development and use of non-dissipativelenergy conserving numerical methods. The opposite is observed for the hybrid approach, for which numerical dissipation plays a key role, and unfortunately is sensitive to the size of the mesh. In particular, when working, the two approaches investigated provide results which are in the same ball range and which agree with what is usually reported in literature. With the hybrid method, however, the inception of instabilities is observed at mesh sizes which vary from case to case, and depend on the propagation model. These results are comforted by numerical computations on a large number of classical benchmarks.
\end{abstract}

\section{Introduction}

The last decades have seen the development of several numerical models allowing the simulation of wave propagation from intermediate depths to shallow water by means of some set of depth averaged Boussinesqtype (BT) equations. Many implementations of these are quite well known in the coastal engineering community, to which they and often freely available. We can for example mention the codes BOUSS-2D [1-4], Funwave [5, 6], Coulwave [7, 8] BOSZ [9], MIKE21 [10], TUCWave [11, 12], and many others. These models allow to accurately simulate the dispersive propagation and shoaling of free surface waves, within some asymptotic error w.r.t. nonlinearity and dispersion parameters depending on wave amplitude, wavelength and depth. The reader may refer to the reviews [13,14] for a broad discussion, and the book [15] for the fundamental aspects concerning the derivation of the underlying partial differential equations. These equations are obtained under the hypotheses of ideal, and most often irrotational flow, and cannot account for the transformation processes taking place in breaking regions. To cope with this limitation, some closure model needs to be introduced. 
At large scales, the main consequence of wave breaking is a strong energy dissipation. So the first attempt to simulate wave breaking was proposed by Zelt [16] introducing a dissipation term in the momentum equation. This term controls the dissipation of energy produced by the wave breaking and it is governed by the value of an eddy viscosity coefficient which must be calibrated with experimental data. Of course, different calibration is needed for different sets of equations. Moreover, to initiate and/or terminate the breaking process some breaking detection criterion needs to be used to activate this eddy viscosity term. The same approach has been followed by many researchers, see for example [17-22]. One of the criticisms to this approach is that, while simple, no direct physical meaning can be attributed to the scaling coefficients involved in the definition of the viscosity [23]. A more relevant physical definition of the effects of breaking on the large scale flow has been attempted using the so-called roller models (see e.g. [23-26]. While based on a better physical background, these models still require some ad-hoc definition of a momentum dissipation, and require some calibration. A more advanced version of these roller models has been proposed in [27], and more recently extended in [28]. These models attempt at accounting for variations along the depth of some of the physical quantities (eddy viscosity, horizontal velocity), thus going beyond the irrotational hypothesis when computing the vorticity and/or dissipation generated in breaking regions. While promising in principle, these models are more complex to implement, require an additional vertical discretization, and have so far been applied only to simple configurations. We also mention the related work presented in $[29,30]$ in which BT models with vorticity effects are discussed. Beside the requirement of a proper calibration of the model, one of the questions we think is not clearly answered in literature is how much the numerical method interacts with the above modelling approaches, and in particular what is the balance between the numerical and model dissipation. This is also related to the fact that almost systematically wave breaking benchmarks are presented without any grid convergence analysis. Another issue is the ability of these approaches to describe properly some special cases as, e.g. stationary hydraulic jumps. To the authors' knowledge there is very little evidence in literature that this type of breaker can be easily modelled with the eddy viscosity approach. Results embedding this type of features, such as e.g. the 2D reef computations presented in [18], again computed on a single mesh. This makes unclear whether the major effect observed is that of the model or that of the limiter/numerical dissipation.

Nevertheless, the eddy viscosity method is a very successful one, which is why we consider its use in this paper. Previous work from one of the authors [12,31] has shown that the classical definition of the eddy viscosity by [17] has trouble detecting stationary hydraulic jumps, and that even modifying the inception mechanisms, the amount of viscosity obtained is not enough for this type of breakers. For this reason we have looked at a more involved approach involving partial differential equations for the main physical quantities: turbulent kinetic energy, energy dissipation, eddy viscosity, and so on. To our knowledge so far only $[1,3,4,32-35]$ have adopted this path, with only [1-4,32,33] actually focusing on complex cases.

As an alternative to the use of eddy viscosity the last ten years have seen the development of a hybrid approach based on a local coupling of the dispersive propagation model with the shallow water equations. It is a simple method in which one first detects breaking regions, and in these the dispersive terms are suppressed. In these breaking regions thus one solves the non-linear shallow water (NSW) equations which allow to model a breaker as a shock. Through this discontinuity mass and momentum are conserved, while total energy is dissipated, thus modelling the energy dissipation due to breaking. Due to its relative simplicity and effectiveness, this approach has gained substantial attention in the coastal engineering community, see for example [6,9, 12,36-38]. The idea was first introduced in [36] in order to exploit the Finite Volume (FV) technique as to simulate discontinuous phenomena such as wave breaking and run-up. In the same work, an indicator criterion for breaking has been extracted based on the similarity between spilling breakers and bores. This criterion has been proven inadequate in some cases [12,39] since its use leads 
to less energy dissipation than needed. Several more sophisticated criteria have been developed based on physical or numerical arguments [12,40-42]. As pointed out in [37], this approach has a major limitation in the stability of the coupling which introduces spurious oscillations at the interface between the breaking and no-breaking region. This phenomenon has been observed by many [43-45], but is unfortunately poorly documented in literature. One of the issues not fully addressed is the role of numerical dissipation in curing this flaw. The use of more robust limiting procedures, is advocated by some as e.g. [43,46] as a means of stabilizing the numerical solution. For example, for of a fully non-linear weakly dispersive propagation model in [43] it is suggested that degrading the accuracy of the numerical discretization in correspondence of the shallow water-Boussinesq interface, thus somehow increasing numerical dissipation, allows to remove numerical perturbations on relatively coarse meshes. Unfortunately, the sensitivity to the grid size for this closure remains unclear. To demonstrate this point we consider the following motivational example involving the shoaling and breaking of a solitary wave on a slope. This example is part of a set of very classical benchmarks by C. Synolakis [47] which we will study in more detail in section $\$$ t. 7.2 . Here, we present results for a breaking case (wave nonlinearity equal to 0.28 ), in which we set manually the transition region according to its known position from the experiments. Compared to actual simulations with the hybrid method, note that this eliminates one of the causes of instability: the intermittency of the detection. We then perform simulations degrading the numerical scheme at the interface between the Boussinesq and

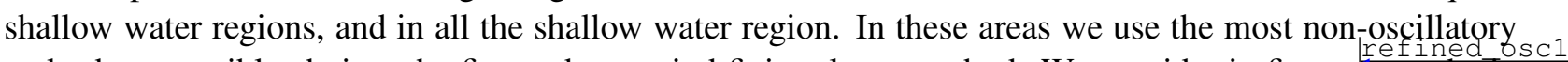
and robust possible choice: the first order upwind finit volume method. We consider in figure 1 results in two very close time instants, on three meshes. The red line shows the detection flag separating the Boussinesq and shallow water regions (one indicates the breaking region, and zero the Boussinesq region). The figures show that: no instabilities whatsoever are observed in the largest gradient region (which will become the bore). An oscillation is instead triggered at the interface, and its blow up is almost instantaneous on the finest mesh, despite the fact that the most dissipative approach available has been used.
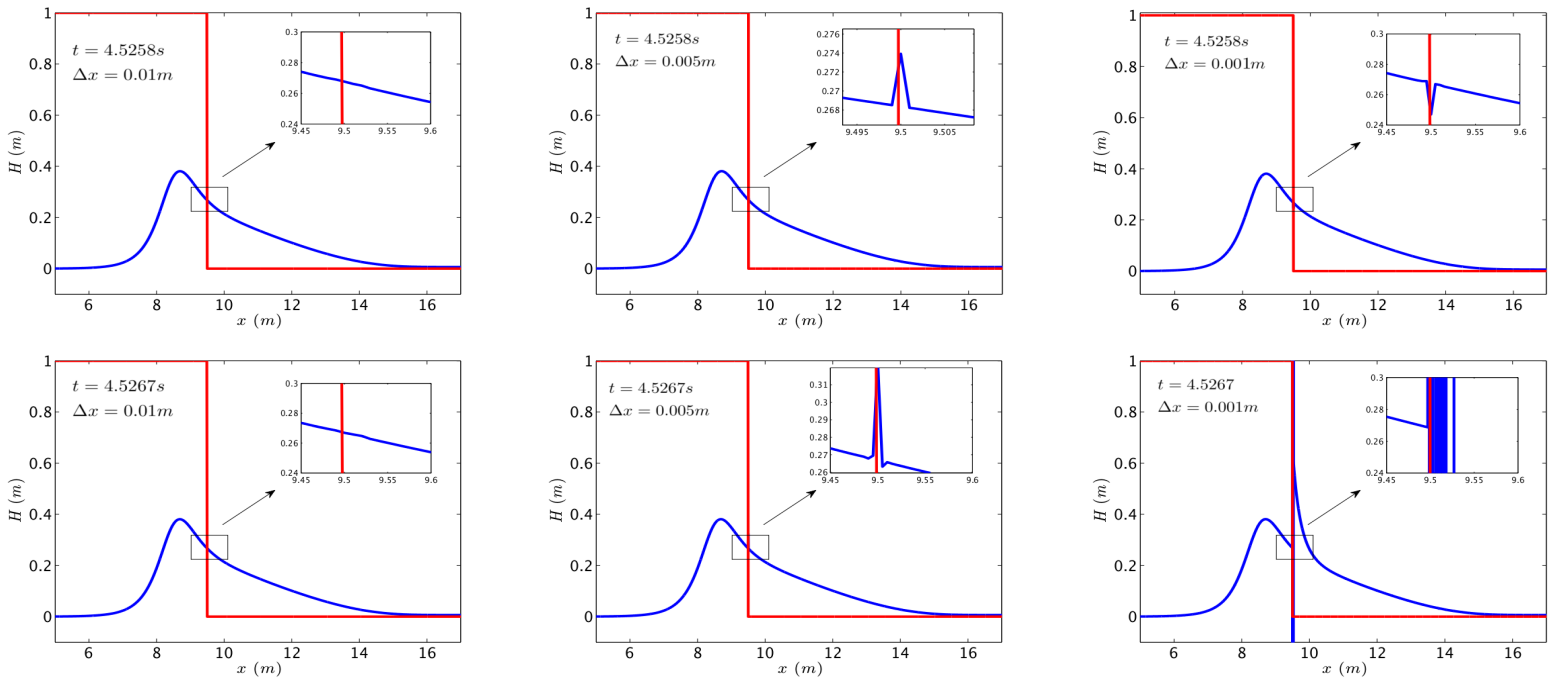

Figure 1: Solitary wave breaking on a slope: hybrid treatment with order reduction at the coupling interface. Wave height at times $t=4.5258 \mathrm{~s}, 4.5267 \mathrm{~s}$ (top and bottom rows), on mesh sizes (from left to right) $\Delta x=0.01 m, 0.005 m, 0.001 m$.

Grid convergence analysis for breaking cases are quite rare in literature. The only exception we are aware of is the single computation shown in [6] in which the authors observe convergence in time aver- 
aged quantities, but report the appearance of increasing oscillations in the pointwise values of the solution without further notice. Clarifying these aspects is of paramount importance. Indeed if one cannot be sure that the mesh size allows the numerical dissipation to be sufficiently large, the initiation of the numerical instabilities may be confused with physical/dispersive effects. A control of this flaw is of course also needed if one wishes for example to use mesh adaptation in breaking regions.

The aim of this work is to investigate the above issues for choices of propagation models and wave breaking closures representative of classical and well known models such as BOUSS-2D [3,4], Funwave [5,6], Coulwave [7, 8], BOSZ [9], MIKE21 [10], TUCWave [11,12], and others. We thus use two enhanced weakly dispersive Boussinesq models: the weakly nonlinear model of Nwogu (used e.g. in BOUSS-2D, BOSZ, and TUCWave), and a frequency enhanced variant of the fully nonlinear Green-Naghdi equations (with similar properties to those used in Funwave and Coulwave). We compare the hybrid approach to an eddy viscosity model. Note that with the eddy viscosity closure the breaking wave fronts are smoother than those obtained with the hybrid method which relies on the approximation of these fronts as shocks. For this reason when using the hybrid approach one has to carefully choose the conservative form used to solve the model, which is essential to recover the right jump conditions, and some form of limiting to avoid the creation of additional spurious numerical oscillations in correspondance of the shock $[11,12,48,60]$.

As mentioned before, the original definition of an eddy viscosity model [17] has been previously shown to have difficulties in handling steady hydraulic jumps [12,31]. For this reason we have chose to use an approach based on the solution of a partial differential equation for the turbulent kinetic energy, similar to the one studied with BOUSS-2D (see [1,3,4,33]). Note that other closures, such as roller models as proposed in $[27,28]$, or other definitions of the eddy viscosity are certainly as valid a choice as the one made here. A thorough comparison of differences in these approaches ins not in our scopes, and is left for future work. Our main objectives are the following:

- to perform a systematic study of the behaviour of the two closures for different mesh sizes, with attention to the possibility of obtaining grid independent results ;

- to gain an insight into the mechanism actually responsible for wave breaking by providing a quantitative description of the different contributions to the dissipation mechanism, differentiating those associated to the numerical scheme from those introduced at the PDE level;

- to provide some understanding of the sensitivity of the above mentioned dissipation to the mesh size;

- to prove the equivalent capabilities of the approaches studied in reproducing simple as well as complex wave transformation, while showing the substantial difference in the underlying dissipation mechanisms.

The paper is organised as follows. Section two presents the two Boussinesq approximations used in this work. Section 3 discusses the numerical approximation of the models, as well as of the wave breaking closure. The comparison of the two approaches on a wide selection of benchmarks is discussed in section 4. The paper is ended by a summary and a sketch of the future and ongoing developments of this work.

\section{Wave propagation models}

\subsection{The weakly nonlinear-weakly dispersive model of Nwogu}

With the notation sketched in figure 2 , we consider the Boussinesq equations proposed by Nwogu [49] based on a weakly-dispersive and weakly-nonlinear asymptotic approximation in terms of the velocity $u^{a}$ 
at an arbitrary distance from a still water level $z^{a}$. Denoting partial derivatives with respect to space and time with the subscripts $x$ and $t$, Nwogu's equations can be cast in a balance law form as follows

$$
\mathbf{U}_{t}+\mathbf{F}\left(\mathbf{U}^{*}\right)_{x}=\mathbf{S}_{\mathbf{b}}-\mathbf{S}_{\mathbf{d}}+\mathbf{S}_{\mathbf{f}}+\mathbf{R}_{\mathbf{w b}}
$$

where $\mathbf{U}$ is the vector of the new variables, $\mathbf{U}^{*}$ is the vector of the conserved variables, and $\mathbf{F}$ is the flux vector

$$
\mathbf{U}=\left[\begin{array}{c}
H \\
P^{*}
\end{array}\right], \mathbf{F}(\mathbf{U})=\left[\begin{array}{c}
H u^{a} \\
H\left(u^{a}\right)^{2}+\frac{1}{2} g H^{2}
\end{array}\right]
$$

The $P^{*}$ variable is a pseudo-mass flux accounting for the vertical (weakly-dispersive and weakly-nonlinear) expansion of the velocity profile:

$$
P^{*}=H u^{a}+H z^{a}\left(\frac{z^{a}}{2} u_{x x}^{a}+\left(d u^{a}\right)_{x x}\right)
$$

In the above equations $d$ denotes the still water depth, $H(x, t)=d(x)+\eta(x, t)$ the total water depth, $\eta(x, t)$ the free surface elevation, $b$ the bathymetry height, $g$ is the gravitational acceleration. As done usually, the value of $z^{a}$ is chosen to optimize the linear dispersion properties of the model, namely $z^{a}=-0.531 d$.

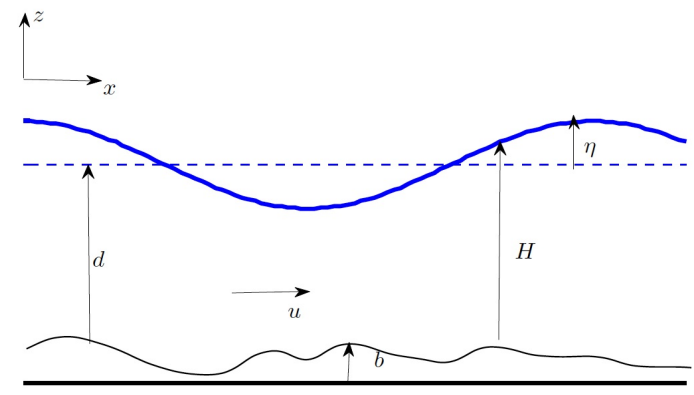

Figure 2: Sketch of the domain.

The three source terms on the right hand side of (11) can be expressed as $\mathbf{S}_{\mathbf{b}}=\left[\begin{array}{ll}0 & -g H b_{x}\end{array}\right]^{T}$, accounting for the effects of the shape of the topography, $\mathbf{S}_{\mathbf{f}}=\left[\begin{array}{ll}0 & -g H S_{f} u^{a}\end{array}\right]$ with $S_{f}=\frac{n_{m}^{2}\|u\|}{H^{4 / 3}}$, accounting for the friction on the bottom, and $\mathbf{S}_{\mathbf{d}}=\left[\begin{array}{lll}\psi_{C} & u^{a} \psi_{C}-\psi_{M}\end{array}\right]$ introduces additional dispersive terms which do not contain time derivatives, and in paticular

$\psi_{M}=-\left(\left(H u^{a}\right)_{x}+\psi_{C}\right) z^{a}\left(\frac{z^{a}}{2} u_{x x}^{a}+\left(d u^{a}\right)_{x x}\right), \quad \psi_{C}=\left[\left(\frac{\left(z^{a}\right)^{2}}{2}-\frac{d^{2}}{6}\right) d u_{x x}^{a}+\left(z^{a}+\frac{d}{2}\right) d\left(d u^{a}\right)_{x x}\right]_{x}$.

The last term on the right hand side is the turbulent wave breaking term, which is only present when this approach is activated. Following $[1,32,33]$ this term has the form

$$
\mathbf{R}_{\mathbf{w b}}=\left[\begin{array}{c}
0 \\
r_{w b}
\end{array}\right]_{x}, \quad r_{w b}=\nu_{t} H u_{x}^{a}
$$

with the eddy viscosity $\nu_{t}$ computed from the discretization of the turbulence model, discussed in $\S 4$ t urb_model 
2. Corrector stage (Adams-Moulton method)

$$
\mathbf{U}^{n+1}=\mathbf{U}^{n}+\Delta t \mathcal{L}^{C} \quad \mathcal{L}^{C}=\frac{9}{24} \mathcal{L}\left(\mathbf{U}^{p}\right)+\frac{19}{24} \mathcal{L}\left(\mathbf{U}^{n}\right)-\frac{5}{24} \mathcal{L}\left(\mathbf{U}^{n-1}\right)+\frac{1}{24} \mathcal{L}\left(\mathbf{U}^{n-2}\right)
$$


with the time step is computed by means of the standard condition $\Delta t^{n+1}=$ CFL $\Delta x / \max _{i}\left(\left|u_{i}^{n}\right|+\sqrt{g h_{i}^{n}}\right)$. Within both stages, the evolution operator $\mathcal{L}$ accounts for all the effects except those of friction and turbulent dissipation (if present). In particular, the shallow water terms are approximated by means of a third order MUSCL finite volume approximation [53,54], with Roe-type numerical fluxes [55]. It is useful for some of the analysis that will follow to report the form of these fluxes reading :

$$
\begin{aligned}
& \mathcal{L}_{i}^{S W}=-\frac{1}{\Delta x}\left(\mathbf{F}_{i+1 / 2}-\mathbf{F}_{i-1 / 2}\right)+\Delta \mathbf{S}_{\mathbf{b} i}^{i+1 / 2}+\Delta \mathbf{S}_{\mathbf{b} i}^{i-1 / 2} \\
& \mathbf{F}_{i+1 / 2}=\mathbf{F}_{i+1 / 2}^{C}-\frac{1}{2}|\mathbf{A}|_{i+1 / 2} \Delta_{i+1 / 2} \mathbf{U}
\end{aligned}
$$

where $\mathbf{F}^{C}$ is the centered flux, and $\Delta(\cdot)_{i+1 / 2}=(\cdot)_{i+1 / 2}^{R}-(\cdot)_{i+1 / 2}^{L}$, and $|\mathbf{A}|_{i+1 / 2}$ is the usual absolute value of the shallow water flux Jacobian, computed via eigenvalue decomposition, and modified with an entropy fix [56,57]. The source term contributions $\Delta \mathbf{S}_{\mathbf{b} i}^{i \pm 1 / 2}$ are well balanced, and involve both a centered and an upwind approximation of the gradient of the bathymetry. We omit details concerning this (quite classical) aspect, for which the interested reader can consult [58-62] and references therein.

Concerning the dispersive terms, the $\mathbf{S}_{\mathbf{d}}$ contribution in (1) is dis discretized using finite differences. While for the hyperbolic component the minimization of the dispersion error requires at least a third order approximation, this is not the case for the higher derivatives in the dispersive terms (see $[5,37]$ for more details on this issue). Here, following [60], the second and third order order derivatives in (1) (1) are treated by means of

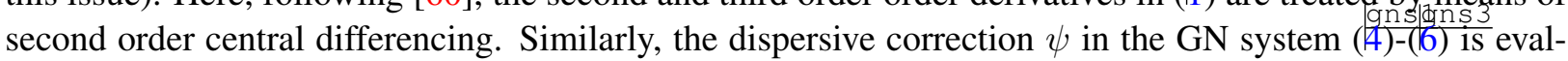
uated by means of a second order $P^{1}$ continuous finite element approximation of the operator $H_{+}+\alpha H \mathcal{T}$ and of the nonlinear forcing temr $\mathcal{Q}$. In absence of friction and turbulent dissipation, equation (b) can be simplified by dividing trough by $H$, and the self adjoint character of $\mathcal{T}$ (equations $\left(\frac{T 1}{7}\right)$ and $\left(\frac{12}{8}\right)$ can be used to deduce a simple variational form reading

$$
\int_{\Omega}\left(v \psi+S_{1}(v) H S_{1}(\psi)+S_{2}(v) H S_{2}(\psi)\right)=\int_{\Omega}\left(S_{1}(v) H S_{1}(w)+S_{2}(v) H S_{2}(w)\right)+\mathrm{Q}(v)
$$

The last expression immediately allows to recover the three diagonal system for the unknown $\psi$ which is symmetric and positive semi-definite. The term $\mathrm{Q}$ on the right hand side is the variational form of the forcing term (9) for which we refer to the full expressions given in [37,63].

The effects of friction and turbulent dissipation (if present) are now embedded in an implicit manner, by appropriately correcting the velocity values. In particular, for Nwogu's equations, the stage iterations (10r) and (III) are modified as follows:

$$
\mathbf{U}^{n e w}-\Delta t \mathbf{S}_{f}^{n e w}-\Delta t \mathbf{R}_{w b}^{n e w}=\mathbf{U}^{n}+\Delta t \mathcal{L}
$$

Accounting for the definitions of the source terms, and of the pseudo mass-flux $P^{*}$ in $(2)$, we obtain the following operator defining the new velocity values (the superscript ${ }^{a}$ is dropped for simplicity)

$$
\begin{aligned}
& H^{\text {new }}\left(u^{\text {new }}+z^{a}\left(\frac{z^{a}}{2} u_{x x}^{\text {new }}+\left(d u^{\text {new }}\right)_{x x}\right)+\Delta t g S_{f}\left(H^{\text {new }}, u^{*}\right) u^{\text {new }}\right)-\Delta t\left(\nu_{t}^{*} H^{\text {new }} u_{x}^{\text {new }}\right)_{x} \\
& =H^{n} u^{n}+H^{n} z^{a}\left(\frac{z^{a}}{2} u_{x x}^{n}+\left(d u^{n}\right)_{x x}\right)+\Delta t \mathcal{L}_{H u}
\end{aligned}
$$

eq:vel_nwogu 
where $\mathcal{L}_{H u}$ is the second component of $\mathcal{L}, u^{*}$ and $\nu_{t}^{*}$ are the last available values of the velocity and turbulent viscosity, and $H_{\text {eq }}^{\text {new }}$ is independently computed from the first discretized equation. As before, the derivatives present in (13) are discretized using second order central finite differences, yielding a tridiagonal system for the new value of the velocity at each stage.

The implementation has been slightly modified for the GN equations. In this case we have added after each of the iterations (10) and (111) a split (in time) implicit discretization of $\mathbf{U}_{t}=\mathbf{S}_{f}^{n e w}+\mathbf{R}_{w b}^{n e w}$. Denoting by $u^{*}$ the last known value of the velocity ( $\operatorname{after}(10)$ and/or (111)), we thus obtain the expression

$$
H^{\text {new }} \frac{u^{\text {new }}-u^{*}}{\Delta t}+g H^{\text {new }} S_{f}\left(H^{\text {new }}, u^{*}\right) u^{\text {new }}=\left(\nu_{t}^{*} H^{\text {new }} u_{x}^{\text {new }}\right)_{x} .
$$

The derivatives in the above expression are then approximated by second order finite differences, leading to a tri-diagonal system again. As in this case the evaluation of the dispersive correction $\psi$ already requires the inversion of a linear system, we have opted here for a simplified implementation involving a few explicit Jacobi relaxation iterations which read

$$
\begin{gathered}
J_{i}^{m}\left(\left(u^{n e w}\right)^{m+1}-\left(u^{\text {new }}\right)^{m}\right)_{i}=-\left(H^{\text {new }} \frac{u^{\text {new }}-u^{*}}{\Delta t}+g H^{\text {new }} S_{f}\left(H^{\text {new }}, u^{*}\right) u^{\text {new }}-\left(\nu_{t}^{*} H^{n e w} u_{x}^{n e w}\right)_{x}\right)_{i}^{m} \\
J_{i}^{m}=\frac{H_{i}^{n e w}}{\Delta t}+g H_{i}^{\text {new }} S_{f}\left(H_{i}^{\text {new }}, u_{i}^{*}\right)+\frac{\left(\nu_{t} H^{\text {new }}\right)_{i+1 / 2}+\left(\nu_{t} H^{\text {new }}\right)_{i-1 / 2}}{\Delta x^{2}}
\end{gathered}
$$

with $H_{i \pm 1 / 2}^{n e w}$ arithmetic averaged values, and with $\left(u^{n e w}\right)^{0}=u^{*}$. Unless otherwise stated, the number of relaxation iterations in the results discussed later has been set to 5 .

Other aspects of the discretizations are related to the modifications of the mass fluxes, velocities, and bathymetry source terms near wet/dry interfaces. Firstly, as in [64], two cut-off values for $H$ are introduced, one to identify dry cells (or nodes), the other to mark as troubled cells (or nodes) in which the division by $H$ may lead to unphysical values of the velocity. To preserve well balancedness in cells containing a dry node, adverse bathymetry gradients are limited as suggested in [59] (see also [64]). In troubled cells (or nodes) instead, the mass flux is set to zero, as well as the velocities, and the dispersive corrections $\mathbf{S}_{\mathbf{d}}$ in (11), the second order terms in (2) and (13), and $\psi$ in (5). The van-Albada slope limiter is used only in breaking regions, and only if the hybrid approach is chosen.

\section{Wave breaking closure}

Boussinesq equations are unable to describe both the overturning of waves, and the dissipation of kinetic energy originated during wave breaking. A physical closure is necessary. Generally, this closure consists of two main steps. The first one is a trigger mechanism allowing to localize in space and time the initiation and the termination of breaking. The second one is a mechanism introducing a dissipation of total energy in the model. This paper focuses on two techniques to define the second element, which are discussed in some detail in the following sections. In both cases, the triggering of wave breaking is done following the criteria proposed $[12,37]$ which have been found simple and robust. The idea is to introduce a flagging strategy based on the following conditions:

- the surface variation criterion: a cell is flagged if $\left|\eta_{t}\right| \geq \gamma \sqrt{g H}$, with $\gamma \in[0.3,0.65]$ depending on the type of breaker; 
- the local slope angle criterion: a cell is flagged if $\|\nabla \eta\| \geq \tan \varphi_{c}$, with critical angle $\varphi_{c} \in\left[15^{\circ}, 30^{\circ}\right]$ depending on the flow configuration.

The first criterion is usually active in correspondence of moving waves and has the advantage of being completely local. The second criterion acts in a complementary manner, and allows to detect stationary or slow-moving hydraulic jumps $[12,18]$. Flagged cells are grouped to form a breaking region . This region is either enlarged to account for the typical roller length, as suggested in [12,65], or deactivated, depending on the value of the Froude number $\mathrm{Fr}^{2}=H_{\max }\left(H_{\max }+H_{\text {min }}\right) /\left(2 H_{\text {min }}^{2}\right)$, defined starting from the minimum and maximum wave height in the flagged zone. The interested reader can refer to $[12,41,65]$ and references therein for mode details regarding the implementation of these detection criteria.

\subsection{Hybrid wave breaking model}

This closure attempts to exploit the properties of hyperbolic conservation laws embedded with an entropy inequality. For the shallow water equations, in particular, the mathematical entropy coincides with the total energy [66-71]. At the continuous level, while conserved in smooth regions, entropy/total energy is dissipated in discontinuous weak solutions. Provided that the numerical scheme introduces the correct amount of dissipation in shocks [69-72], this lends itself naturally for the wave breaking closure, This approach is in itself neat and simple. It has the limitation that the form of the dissipation is, at best, fixed by that determined by the shallow-water Rankine-Hugoniot jump conditions. This quantity can be analytically computed and it is given by (see e.g. [73] chapter 1.6, and [74])

$$
\mathcal{D}_{\mathrm{sw}}=g \sqrt{g \frac{H_{\max }+H_{\min }}{2 H_{\max } H_{\min }}} \frac{\left(H_{\max }-H_{\min }\right)^{3}}{4}
$$

This is however a parameter free definition of the dissipation which has been proved to reproduce quite well the large scale decay of the total energy in for several types of breaking waves, and with several different underlying propagation models and relative numerical discretizations $[6,12,36-39,41,75]$. The implementation of this closure is somewhat trivial once the wave detection algorithm discussed earlier has been properly set up. For the Nwogu's equations, it boils down to locally turning off in the whole flagged region the dispersive source $\mathbf{S}_{\mathbf{d}}$ and the second order derivative terms in (13) when evaluating the new nodal velocities. Similarly, for the GN system, the nodal values of $\psi$ in (g) are set to zero in the breaking region.

The most limiting aspect of this approach is the switch between the non-hydrostatic and the hydrostatic equations. What has been reported by many authors in a more or less marked way, is the difficulty of performing this switch in a stable manner. Unless coarse grids are considered, with eventually the addition of local regularization numerical dissipation terms, several authors have reported the appearance of strong oscillations $[6,12,37,43]$. These artefacts tend to become stronger and stronger as the mesh is refined. To our knowledge, there are no studies in literature reporting fully grid converged solutions with this approach due to this problem. An exception to this is perhaps one result reported in [6] showing some convergence (on only 3 grids) of the time averaged wave heights and setup, even though increasing oscillations in the local profiles are reported for the same test. This behaviour clearly poses a limitation in terms of potential for local automatic adaptation of the mesh, and its investigation is one of the objectives of this article.

\subsection{Eddy viscosity closure via a PDE based TKE model}

The use of an eddy viscosity model to provide the dissipation required for the breaking closure is one of the earliest approaches [16]. The definition of this artificial viscosity is the key of this approach, as well as the way in which it enters the Boussinesq equations. On of the most common approaches, due to Kennedy 
and collaborators [17] (see also [12,18,22,31] and references therein), involves a definition of the eddy viscosity based essentially on the variation in time of the free surface elevation. This term is then embedded in a viscous flux, as e.g. in (1/) and (I) richer physical description of the vertical kinematics and of the effects of turbulence (e.g. the so-called roller models). Some approaches explicitly embed the effects of the dynamics of vorticity (roller-models) [27,28], others include partial differential equations for an average turbulent kinetic energy $[1,33]$, and other introduce a multi-layer description embedding PDEs for a turbulent layer flowing on top and interacting with the bulk of the wave, well representative of spilling flows [34,35,76-78]. Simpler methods have attempted at improving the behaviour of the total energy dissipation by also including a water elevation viscosity [23]. In this work, we have chosen to adopt a model of intermediate complexity based on the solution of an additional PDE, weakly coupled to the main Boussinesq system of equations. In particular consider the approach initially proposed by Nwogu [1] who used a standard TKE (turbulent kinetic energy) equation coupled to he fully non-linear equations of Wei et al. [79]. A highly non-linear Boussinesq model with the same turbulence wave breaking model of Nwogu has been used by Elnaggar and Watanabe [32]. More recent work on the same model is discussed in [33] where the TKE equation is manipulated to obtain a PDE for the eddy viscosity which is coupled to a fully nonlinear fully dispersive Green-Naghdi model. Here we propose a variant of the model proposed by Nwogu modified according to some of the definitions proposed in [33], as well as some definition which improve the consistency of the model with the wave breaking detection criteria we adopt.

Following $[33,80]$, the eddy viscosity is determined from the amount of the turbulent kinetic energy $k$, produced by the wave breaking, and a turbulent length scale $\ell_{t}$ :

$$
v_{t}=C_{\nu} \sqrt{k} \ell_{t}
$$

In $k-L$ turbulence models [81,82] (see also [33]), the constant $C_{\nu}$ is usually set to $C_{\nu}=(0.09)^{1 / 4} \approx 0.55$ which is the value used here. We now need a model for the computation of $k$ and $\ell_{t}$. Differently from the models discussed in $[81,82]$, here we adopt a one equation approach in which only one PDE is solved for $k$, while the for $\ell_{t}$, inspired by the definition used in [33], we use a vertical average mixing length defined as

$$
\ell_{t}=\kappa H
$$

where $\kappa$ is a constant controlling the width and intensity of the breaking. The length $\ell_{t}$ is expected to be of the order of the wave height [1], so $\kappa$ is a case dependent constant. Concerning turbulent kinetic energy, it can be shown that in three space dimensions the following transport equations holds [80]

$$
k_{t}+\mathbf{u} \cdot \nabla k=\mathcal{D}+\mathcal{P}-\mathcal{E}
$$

with $\mathcal{D}, \mathcal{P}$, and $\mathcal{E}$, diffusion, production and dissipation (or destruction) terms respectively. Definitions and possible expressions of these quantities in terms of mean flow quantities can be found e.g. in the book [80].

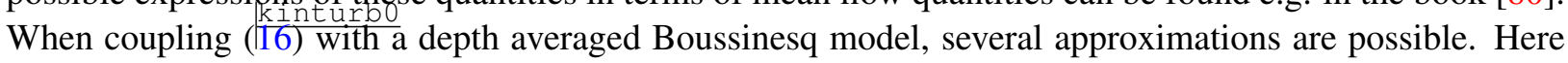
we will combine some of the elements suggested in [1] and in [33] in order to obtain a model simple to implement, to be compared to the hybrid approach. First of all, we will assume that both $k$ (and hence $\nu_{t}$ ) and its transport dynamics are constant along the depth, so that (16) can be replaced by a zero-th order approximation involving only depth averaged quantities, namely

$$
(H k)_{t}+(H u k)_{x}=H \overline{\mathcal{D}}+H \overline{\mathcal{P}}+H \overline{\mathcal{E}}
$$


For the definition of the terms on the right hand side of (17) we have followed [1]. In particular, we have for the diffusion and destruction terms

$$
H \overline{\mathcal{D}}=H \sigma \nu_{t} k_{x x}, \quad H \overline{\mathcal{E}}=-H C_{D} \frac{k^{3 / 2}}{\ell_{t}}
$$

where, following [1,33], we have set $C_{D}=C_{\nu}^{3}$. The constant $\sigma$ allows to control the smoothness of the TKE, and hence of the brea?king viscosity, in the breaking region. Concerning the production term, the model used is again the one suggested in [1] assuming this quantity to depend on the vertical gradient of the velocity at the free surface. Following the notation of (16), and denoting the velocity at the free surface by $\mathbf{u}^{s}=\mathbf{u}(t, x, y, z=\eta)$, we have

$$
H \overline{\mathcal{P}}=H B(t, x) \mu_{\mathcal{P}} \mathbf{u}_{z}^{s} \cdot \mathbf{u}_{z}^{s}
$$

As in [1], the turbulent viscosity $\mu_{\mathcal{P}}$ appearing in the production term is defined based on a mixing length hypothesis assuming a balance between production and dissipation, namely

$$
\mu_{\mathcal{P}}=\frac{\ell_{t}^{2}}{\sqrt{C_{D}}} \sqrt{\mathbf{u}_{z}^{s} \cdot \mathbf{u}_{z}^{s}}
$$

so that we end with

$$
H \overline{\mathcal{P}}=H B(t, x) \frac{\ell_{t}^{2}}{\sqrt{C_{D}}}\left(\mathbf{u}_{z}^{s} \cdot \mathbf{u}_{z}^{s}\right)^{3 / 2} .
$$

In [1] the parameter $B$ is equal to 0 or 1 depending on a wave breaking criterion. In the reference the criterion used is based on the ratio between the free surface velocity and the wave celerity being larger than one. Here, for simplicity $B$ is set to one in the breaking regions detected exactly as discussed in the beginning of section $\delta 4$. This also allows to detect wave breaking in the same way for the TKE and hybrid approach. Having fixed the values of $C_{\nu}$ and $C_{D}$, the only "tunable" parameters are $\kappa$ and $\sigma$.

Lastly, we need to be able to evaluate the depth averaged and free surface velocities for both Boussinesq models, as well as the value of the vertical gradient of the velocity at the free surface. For this we use the vertical asymptotic development underlying the two models. In the weakly nonlinear case, this development can be used to write the following relations $[15,49]$

$$
u(z)=u^{a}-\left(\frac{z^{2}}{2}-\frac{d^{2}}{6}\right) u_{x x}^{a}-\left(z+\frac{d}{2}\right)\left(d u^{a}\right)_{x x}
$$

giving the free surface vertical gradient

$$
u_{z}^{s}=-\eta u_{x x}^{a}-\left(d u^{a}\right)_{x x} .
$$

and the depth averaged (within the asymptotic accuracy) velocity required for the transport term in (1Tintur

$$
u=u^{a}+\left(\frac{\left(z^{a}\right)^{2}}{2}-\frac{d^{2}}{6}\right) u_{x x}^{a}+\left(z^{a}+\frac{d}{2}\right)\left(d u^{a}\right)_{x x}
$$

ubar_ua

The GN equations directly provide a value of the depth averaged speed, while the fully nonlinear asymptotic development allows to write

$$
u(z)=u-\left[\frac{z^{2}}{2}-\left(\frac{H^{2}}{6}-\frac{H(H-d)}{2}\right)\right] u_{x x}-\left[z-\left(\frac{H}{2}-d\right)\right](d u)_{x x}
$$


which yields a similar expression for the vertical gradient of the free surface velocity, this time in function of the depth averaged velocity $u$ :

$$
u_{z}^{s}=-\eta u_{x x}-(d u)_{x x} .
$$

The fully discrete distribution of the nodal values of the TKE is obtained by integrating equation (IT) with a semi implicit approach. Before the predictor step (10) is applied to the Boussinesq models, the nodal TKEs are evolved by first applying an explicit Euler update involving a third order MUSCL upwind discretisation of the transport operator $(H u k)_{x}$, essentially the same presented in section $\S 3$ for the shallow water equations. To avoid spurious negative values in this phase, the min-mod limiter is applied [83]. The predicted values $k_{i}^{*}$ are then corrected by means of diagonally semi-implicit relaxation iterations similar to those used for the breaking dissipation and reading

$$
\begin{aligned}
\left(\frac{\Delta x}{\Delta t}+\frac{2 \sigma v_{t, i}^{n}}{\Delta x}\right)\left(k_{i}^{m+1}-k_{i}^{m}\right) & =\Delta x \frac{k_{i}^{m}-k_{i}^{*}}{\Delta t}+\sigma v_{t, i}^{n} \frac{k_{i+1}^{m}-2 k_{i}^{m}+k_{i-1}^{m}}{\Delta x} \\
& +\left(\frac{B \ell_{t, i}^{2}}{\sqrt{C_{D}}}\left(u_{z}^{s}\right)_{i}^{3 / 2}\right)^{n}-C_{D}\left(\frac{k_{i}^{3 / 2}}{\ell_{t, i}}\right)^{n}
\end{aligned}
$$

with an initial condition, $k^{0}=k^{*}$. For the benchmarks discussed in the paper, 4 or 5 relaxation iterations are used unless otherwise stated. Where necessary, depth average velocity (for the Nwogu model) and velocity gradient at the free surface (for both Boussinesq models) are obtained by a second order central finite difference approximation of (21), (20), and (22).

As a final note, we will keep in the following the notation TKE when referring to this closure, as this eddy viscosity method clearly relies on the solution of the PDE for the turbulent kinetic energy.

\section{A note on the dissipation mechanisms at work}

One of the key aspects concerning the numerical modelling of wave breaking is the notion of dissipation. As discussed in the introduction, the mechanisms related to the transformation of potential energy into mechanical energy, and its subsequent dissipation, are not embedded in Boussinesq models that we study which stem from a potential description of the flow. The role of the closure model is thus to mimic these mechanisms. Clearly the main interest in the closure is to be able to predict correctly the dynamics of wave heights and (in the multidimensional case) currents. It is however interesting to understand what is the underlying dissipation mechanism active during the numerical breaking process. The main question we want to contribute to answer to in this paper is how much the numerical method is involved in this process, and if it is at all. We provide here a short discussion of this aspect, and suggest quantities which we will use in the numerical applications to quantify the contributions to the breaking process of the numerics, as well as of the PDE model itself.

A proper formulation of this analysis requires a formal definition of what is the energy to be dissipated for the propagation models under consideration. This has to be done at the continuous level, but of course we must be able to provide an appropriate discrete translation of this energy conservation/dissipation statement. We recall that the PDE systems used in this paper have been chosen as representatives of models/codes well known the community such as BOSZ [9], MIKE21 [10], BOUSS-2D [3,4], TUCWave [11, 12], Funwave $[5,6]$, Coulwave [7,8]. Unfortunately, while the GN equations do have a total energy which one may choose to use for this purpose, this is not the case for Nowgu's model. For the latter one can only derive 
A consequence of this discussion is that an exact evaluation of the dissipative mechanisms for the type of models used here is not within our grasp. So, in order to be able to provide some quantitative information on the sources of dissipation, we had to make some choices, and some hypotheses. We start by recasting our PDE models as the shallow water system plus a dispersive source

$$
\partial_{t} \mathbf{U}+\partial_{x} \mathbf{F}(\mathbf{U})-\mathbf{S}_{b}-\mathbf{S}_{f}-\mathbf{R}_{w b}=\mathcal{D}
$$

This is a form similar to (11), except that in the above equation the left hand side only contains the shallow water terms and the eddy viscosity model, if present. All the dispersive terms are included in $\mathcal{D}$. We then look at the contributions to the balance of the shallow water total energy, whose time variation can be expressed as (see e.g. [67-69])

$$
\partial_{t} E=\mathbf{V}^{t} \partial_{t} \mathbf{U}=-\mathbf{V}^{t}\left(\partial_{x} \mathbf{F}(\mathbf{U})-\mathbf{S}_{b}-\mathbf{S}_{f}-\mathbf{R}_{w b}-\mathcal{D}\right)
$$

with $E=H\left(g H+u^{2}\right) / 2+g H b$, and having denoted by $\mathbf{V}^{t}$ the transpose of the array of the so-called energy (or entropy, or symmetrizing) variables $\mathbf{V}^{t}=\left[g \eta-u^{2} / 2, u\right]$. For both numerical models tested in the paper, we can easily provide a nodal discrete analog of the last expression which, using the notation of (12), reads

$$
\partial_{t} E_{i}=\mathbf{V}_{i}^{t} \partial_{t} \mathbf{U}_{i}=-\mathbf{V}_{i}^{t}\left(-\mathcal{L}_{i}^{S W}-\mathbf{S}_{f i}-\mathbf{R}_{w b i}-\mathbf{D}_{i}\right)
$$

Neglecting the boundary conditions (or assuming periodic or null the boundary fluxes), the total variation of the shallow water energy can be deduced using the explicit form $\mathcal{L}_{i}^{S W}$ and of the central and upwind contributions in the bathymetry terms [58-62]. The final result can be recast as

$$
\sum_{i \geq 1} \Delta x \partial_{t} E_{i}=\sum_{i \geq 1} \Delta x \mathbf{V}_{i}^{t} \partial_{t} \mathbf{U}_{i}=\sum_{i \geq 1} \Delta \mathcal{F}_{i}^{E}-D_{\text {upwind }}-D_{\text {friction }}-D_{\text {vis }}+\Delta E_{\mathcal{D}}
$$

We can now try to say more on the terms on the right hand side. It seems quite reasonable to assume that wave breaking is not associated to the dispersive contributions. This means that we will leave out of the analysis the contributions of the dispersive source $\Delta E_{\mathcal{D}}=\sum_{i} \mathbf{V}_{i}^{t} \mathcal{D}_{i}$. Another term which in principle 
one would expect not to contribute to the analysis, is the centered part of the flux which enters the above expression via the terms

$$
\Delta \mathcal{F}_{i}^{E}=-\mathbf{V}_{i}^{t}\left(\mathbf{F}_{i+1 / 2}^{C}-\mathbf{F}_{i+1 / 2}^{C}+\frac{g H_{i+1 / 2}}{2} \Delta_{i+1 / 2}[0, b]^{t}+\frac{g H_{i-1 / 2}}{2} \Delta_{i-1 / 2}[0, b]^{t}\right) .
$$

This is where the analysis provided in e.g. $[69,70]$ is most useful. Without going into much detail, the references provide a very simple rule to define the centered flux for which one can show that $\Delta \mathcal{F}_{i}^{E}=$ $\mathcal{H}_{i+1 / 2}-\mathcal{H}_{i-1 / 2}$, with $\mathcal{H}_{i \pm 1 / 2}$ consistent numerical approximations of the total energy flux. This algebraic relation leads to the conclusion $\sum_{i \geq 1} \Delta \mathcal{F}_{i}^{E}=0$ exactly, whether the solution is continuous or not. This means that, even if slightly different implementations of the central flux are used, this quantity is in principle not relevant for our analysis.

This leaves three quantities to be monitored, associated to the numerical (upwind) dissipation

$$
D_{\text {upwind }}=\sum_{i \geq 1}\left\{\Delta \mathbf{V}_{i+1 / 2}^{t}\left(\frac{1}{2}|\mathbf{A}|_{i+1 / 2} \Delta_{i+1 / 2} \mathbf{U}+\frac{g H_{i+1 / 2}}{2} \operatorname{sign}(\mathbf{A})_{i+1 / 2} \Delta_{i+1 / 2}[0, b]^{t}\right)\right\}
$$

and to the friction and wave breaking (eddy viscosity) model

$$
D_{\text {friction }}=\sum_{i \geq 1} g H_{i} S_{f i} u_{i}^{2} \quad \text { and } \quad D_{\text {vis }}=\sum_{i \geq 1} \nu_{t, i+1 / 2} H_{i+1 / 2}\left(\Delta_{i+1 / 2} u\right)^{2} .
$$

Note that with the spatial discretization choices made both $D_{\text {friction }}$ and $D_{\text {vis }}$ are clearly positive definite. The same cannot be said a-priori about the upwind dissipation $D_{\text {upwind }}$. To be sure of the positivity of this term, indeed one should have implemented the dissipation in terms of variations of the entropy variables [69-71], instead of using in the numerical flux variations at cell interfaces of the conservative variables, as done in standard implementations of the upwind flux. Furthermore, this term involves both the reconstruction and the limiter, the latter only in the shallow water regions associated to wave breaking when using the hybrid approach of section $\S 4.1$. This makes it a perfect candidate to monitor the impact of the numerical choices and their contribution to the wave breaking process, and when possible compare these contributions to those of the eddy viscosity and friction terms. This analysis has been performed for three of the benchmarks proposed, involving both periodic and non periodic waves, dry areas, as well as pure propagating bores. Note that in practice the above expressions have to be evaluated in post-processing, by saving the different terms evaluated during the computations. The time stepping of course also plays a role in this analysis. The interested reader can refer to [72] for a discussion on this. To minimize these effects, while keeping as much as possible of the actual terms computed in the code and used to obtain the numerical solutions, in all the cases presented we have used $t^{n+1 / 2}$ half time-step evaluations of these terms by averaging values at $t^{n}$ and $t^{n+1}$.

\section{Boundary condition and the internal source function}

In this work we use two types of boundary conditions : solid (reflective) wall and absorbing boundary conditions. For the wall boundary conditions ghost cells are used with mirrored states for the velocities, as discussed in [60]. Absorbing boundaries are used for outgoing waves. In this case, an adsorbing layer is introduced within which surface elevation and the momentum are damped by multiplying their values by a coefficient $m(x)$ defined as [12]

$$
m(x)=\sqrt{1-\left(\frac{d(x)}{L_{s}}\right)^{2}}
$$


where $L_{s}$ is the sponge layer width, and $d(x)$ is the distance from the end of the absorbing boundary. As prescribed in [12], the width $L_{s}$ should depend on the wavelength of the outgoing wave. For a given wavelength $L$, the sponge layer width should be $L \leq L_{s} \leq 1.5 L$.

Concerning wave generation, we follow the approach of Wei et al. [90]. To obtain a desired oscillation signal in the wave generating area, a source function $S(\mathbf{x}, t)$ is added into the mass conservation equation at each time step, which is expressed as

$$
S(\mathbf{x}, t)=D^{*} \exp \left(\gamma\left(x-x_{s}\right)^{2}\right) \sin (-\omega t)
$$

in which

$$
\gamma=\frac{5}{(\delta L / 4)^{2}}=\frac{80}{\delta^{2} L^{2}}
$$

where $L$ is the wave length, $\omega$ the wave frequency, $\theta$ the wave incident angle, $x_{s}$ is the location of the center of the wave-making area, $\delta$ is a parameter that influences the width $W=\delta L / 2$ of the wave generator area and $D^{*}$ is the source function's amplitude. For a monochromatic wave, $D^{*}$ is defined as

$$
D^{*}=\frac{2 \sqrt{\gamma} A_{0}\left(\omega^{2}-\alpha_{1} g \mathrm{k}^{4} h^{3}\right)}{\omega \mathrm{k} \sqrt{\pi} \exp \left(-l^{2} / 4 \gamma\right)\left[1-\alpha(\mathrm{k} h)^{2}\right]}
$$

where $h$ is the still water level at the wave generation region, $A_{0}$ the wave amplitude, $l\left(=\mathrm{k}_{x}\right)$ the wave number in the $x$-direction, $\alpha=-0.390$ and $\alpha_{1}=\alpha+1 / 3$.

\section{Numerical results}

\subsection{Wave breaking over a bar}

This test case of Beji and Battjes [91] examines the sinusoidal wave propagation over a submerged bar. The scope of this test case is to investigate the frequency dispersion characteristic and non-linear interaction of complex wave propagation phenomena. A sketch of the problem is provided in figure 3 . The computational domain is $x \in[0,35 m]$, with sponge layers placed at both ends. Periodic waves were generated at $x=$ $10 \mathrm{~m}$ over a mean water depth of $0.4 \mathrm{~m}$. Wave height and period are set to $a=0.054 \mathrm{~m}$, and $T=2.5 \mathrm{~s}$, corresponding to a dispersion parameter $k h \approx 0.52$. Waves propagate over submerged trapezoidal bar with a toe at $x=15 \mathrm{~m}$, a front slope of $1: 20$, a $2 \mathrm{~m}$ long plateau of $0.3 \mathrm{~m}$ height, and a lee slope of $1: 10$. More informations on the experiment can be found in [91] and in the references using this test case for model validation $[12,37,65,92]$.

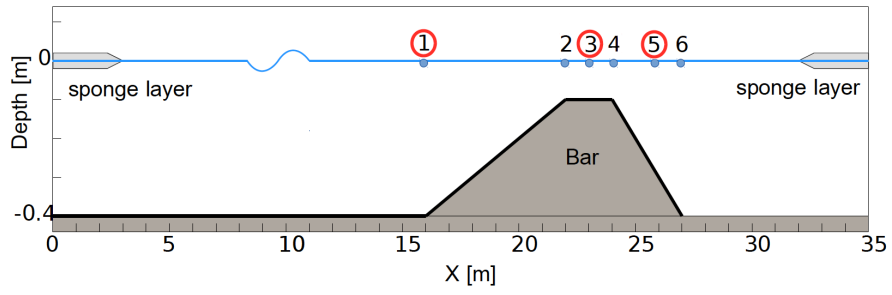

Figure 3: Wave breaking over a bar: problem sketch, and position of the gauges 
Concerning the model parameters, for this highly unsteady problem the surface variation detection parameter $\gamma$ (cf. section $\S 4$ ) is the one more sensitive to the onset of breaking. For the computations performed here we have set $\gamma=0.3$. The parameters used for the TKE are not the same for the two Boussinesq propagation models. In particular, we have set $\kappa_{G N}=2.8$ and $\sigma_{G N}=1.2$ for the GN equations, while $\kappa_{N}=3.2$ and $\sigma_{N}=1.2$ for the Nwogu system.

Experimental data are available in several wave gauges placed before, on top, and after the bar. Here we focus on three gauges (cf. figure 3 ) placed before the toe of the bar, gauge 1 at $x=16 \mathrm{~m}$, on top of the plateau, gauge 3 at $x=23 \mathrm{~m}$, and on the lee slope, gauge 5 at $x=26 \mathrm{~m}$. We will discuss numerical results obtained on three different meshes of size $4 \mathrm{~cm}, 2 \mathrm{~cm}$, and $1 \mathrm{~cm}$. For the Nwogu model, we could not run the hybrid breaking simulations on the last mesh due to instabilities at the Boussinesq-shallow water interface. Similarly, when using the hybrid approach we could not go below $\Delta x=1 \mathrm{~cm}$ when using the GN model for propagation. Note also that the results discussed here are those obtained after a transient of 36 seconds, differently from what is done e.g. in $[12,37]$, where the four first waves are analyzed. The results

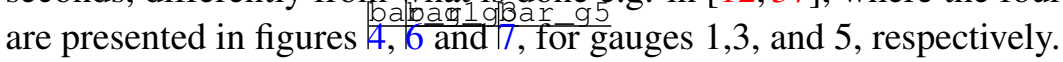

Figure $\frac{\text { bar } \frac{}{4} \text { allows }}{4}$ to visualize the behaviour of the models at the toe of the bar, right at the end of the wave propagation region. This gauge allows to highlight the initial asymmetry of the waves, essentially due to the interaction with the submerged bar. Some preliminary observations can be made. Firstly, the fully nonlinear model (left column) seems to capture better the shape of the waves, the weakly nonlinear one providing a signal which is slightly too peaky. Secondly, we see already at this stage that while the TKE model (blue curves) shows little sensitivity to the mesh size, the signals obtained with the hybrid approach (green curves) depend strongly on this parameter. We can clearly see on the intermediate and fine mesh (in the GN case) higher frequency components absent in the TKE results. These components are generated in correspondence of the boundary of the wave breaking region, as it can be clearly seen in the snapshots of figure 5 . These instabilities become stronger as the mesh is refined, and may ultimately lead to the blow up of the solution, as it is the case for the Nwogu model on the fines mesh, and of the GN model on finer meshes.

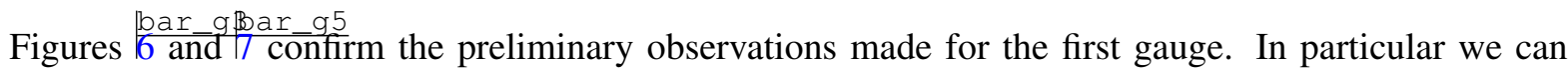
clearly see the strong dependence of the results of the hybrid model on the mesh size. For this approach we can also see how the breaking waves are represented as very sharp fronts. For the GN model, on the coarse mesh breaking stops early enough for the signal in these two gauges to be smooth. This however leads to a noticeable phase lag. As the mesh is refined, the waves break more strongly. This leads clearly to an improvement on the phase. This behaviour curiously is not observed for the Nwogu model which shows strong and sharp breaking fronts already on the coarsest mesh level, with a correct phase. This allows to highlight the need of tailoring the choice of the breaking detection criterion to the propagation model. Here the same parameters have been used for both. Nevertheless, both set of results allow to visually see the appearance of spurious higher frequencies in the signal. These are the result of the coupling between the dispersive and non-dispersive regions. For the weakly non-linear model (right column) we can see the inception of the instability already on the medium resolution used here in figure $\frac{\text { bar }}{7 .}$ This is less evident for the GN model, which still provides numerical solutions on the finer level used. We where however unable to refine once more the mesh without solution blow up.

The TKE approach is clearly less sensitive, at least for this test, to both the choice of the model parametrization, and the mesh size. This is summarized in figure 8 , showing a grid convergence for the gauge 3. We also would like to remark that, for Nwogu's equations and for plunging breakers, Demirbliek and Nwogu in [3] resorted to a more complex TKE closure with a PDE for the $B$ coefficient in the production term (19). We found out that the simplified formulation adopted here, combined with the physical 

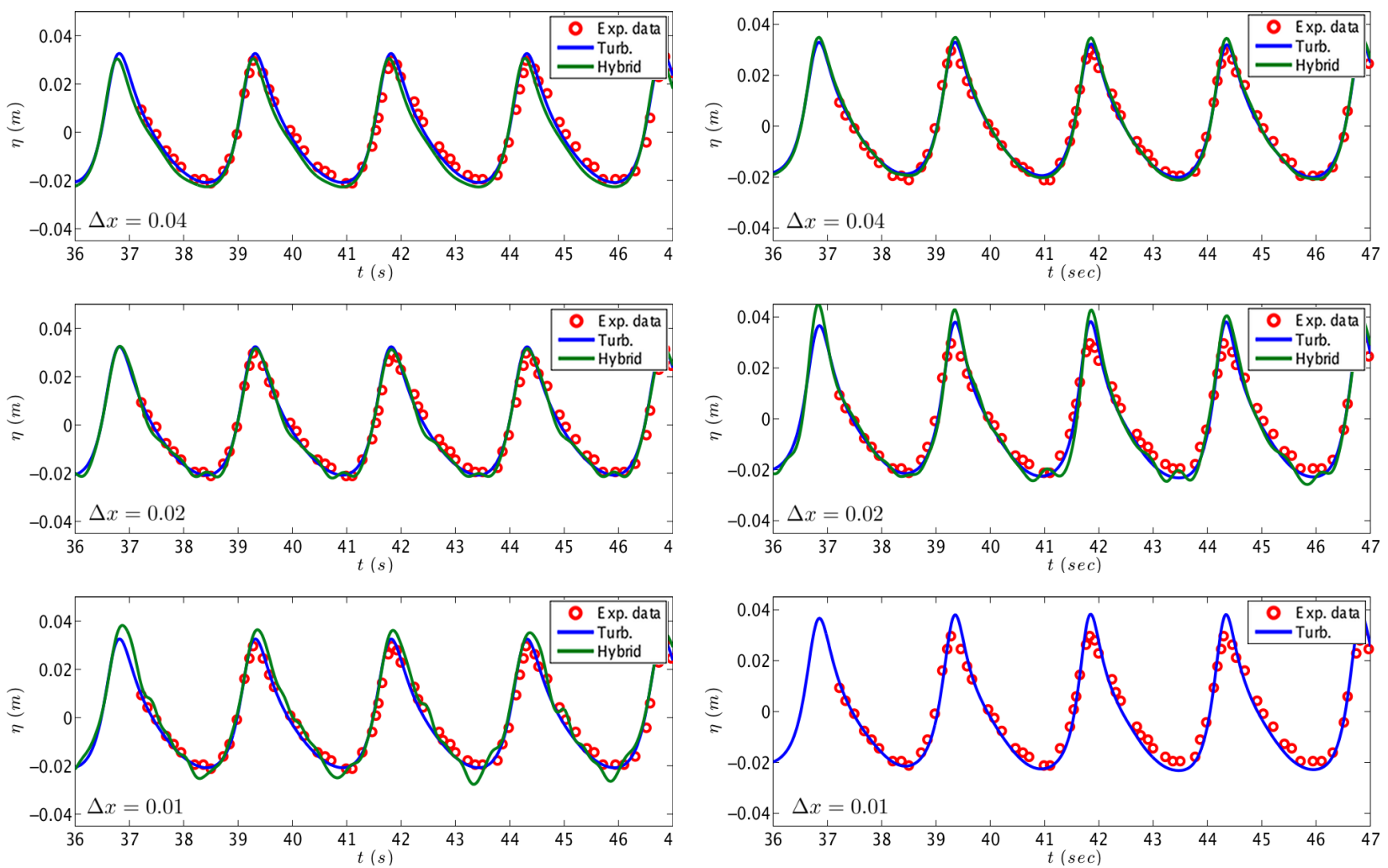

Figure 4: Time series of surface elevation at wave gauge 1 for the GN (left) and Nwogu (right) models using the TKE (blue) and Hybrid (green) wave breaking closure. Mesh size is $0.04,0.02,0.01 \mathrm{~m}$ from top to bottom.
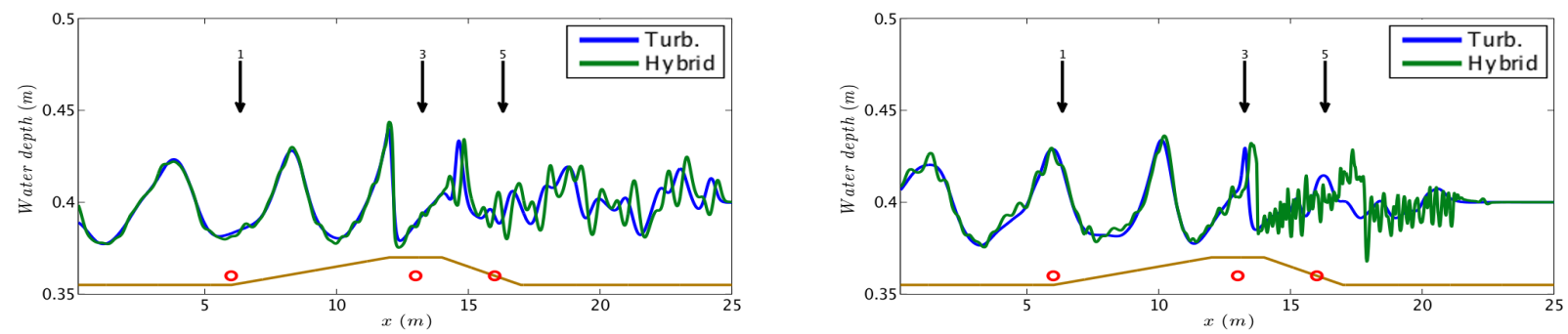

Figure 5: Snapshots of the flow for the GN (left) and Nwogu (right models) using the TKE (blue) and Hybrid (green) wave breaking closure. Mesh size is $0.02 \mathrm{~m}$

criteria for the initiation and termination of the process discussed in the beginning of section $\S 4$ can simulate reasonably well plunging wave breakers.

\subsubsection{Dissipation mechanisms}

We report in figures 9 and 9 and the time evolution of the dissipation terms active for this test (cf. section $\S$ ): $D_{\text {upwind }}$ (in blue) and $D_{\text {vis }}$ (in green). The flow is periodic so we focused on 5 periods from time $12 \mathrm{~s}$ to time $14 \mathrm{~s}$. The results show the dissipation flashing when the tallest wave approaches the bar, and then 

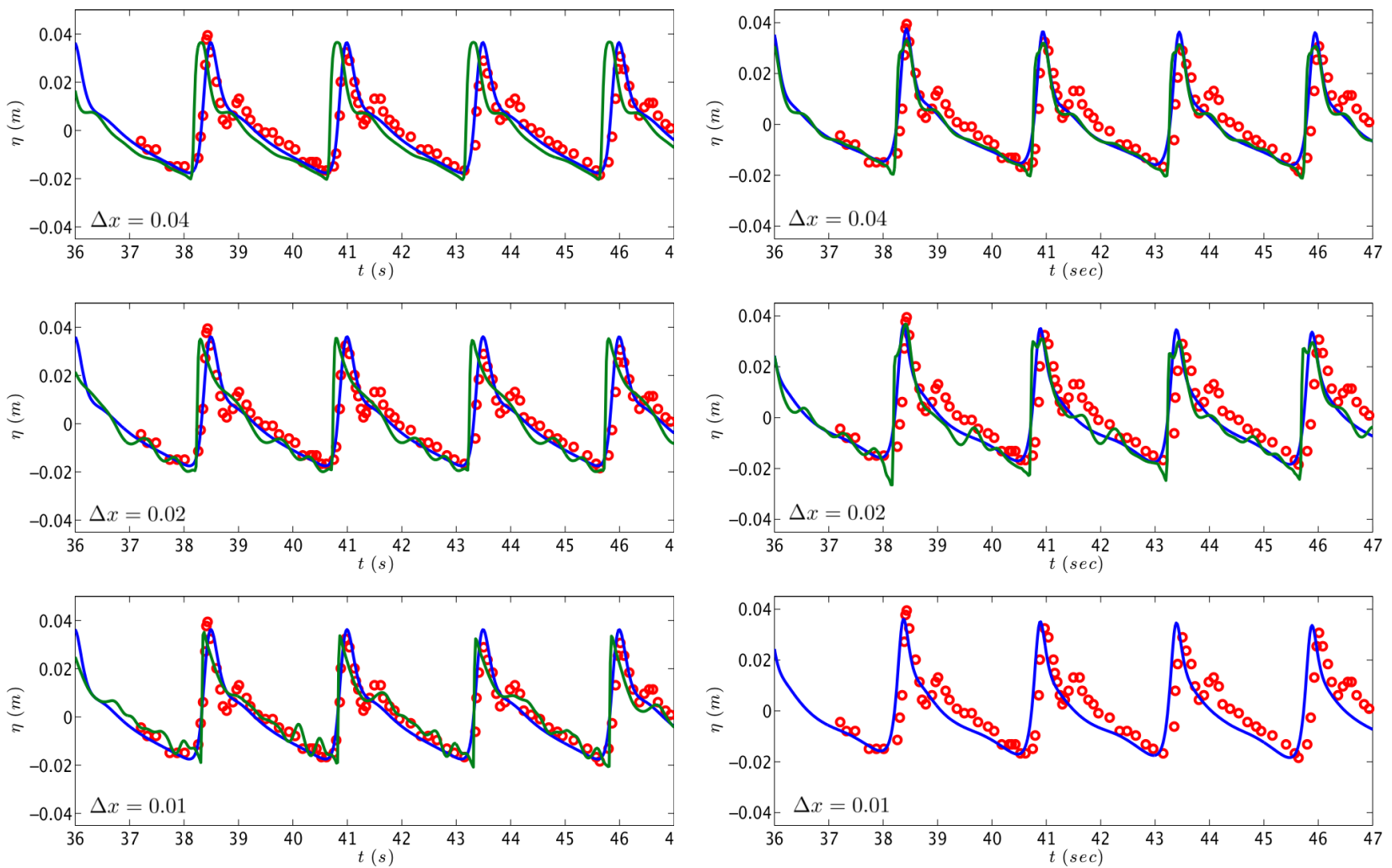

Figure 6: Time series of surface elevation at wave gauge 3 for the GN (left) and Nwogu (right) models using the TKE (blue) and Hybrid (green) wave breaking closure. Mesh size is $0.04,0.02,0.01 \mathrm{~m}$ from top to bottom.

reducing as the breaking process continues on top of the bar. Also, the inception of breaking for the Nwogu model has a phase advance of about one second which can be explained by the over-shoaling characteristics of this model [93,94]. The results for the GN model, figure 9, allow the following remarks. The role of numerical dissipation $D_{\text {upwind }}$ when using the eddy viscosity closure (left column) is extremely small. This term definitely does not contribute at all to the breaking process. On the second mesh, its values approach machine zero. On the contrary, in the case of the hybrid closure, $D_{\text {upwind }}$ is doing all the job. We can also see that the on the coarser mesh the area under the dissipation bells is larger, which means that the overall contribution in time to the energy dissipation is more important. When using the model of Nwogu, figures 10 , we can see again, from the left column, that the numerical dissipation plays no role in the breaking process, and it quickly reaches very low values. The right column allows to visualize the inception of the numerical instabilities (top figure) and their blow up (bottom figure). Note that for the finer meshes used in figure 8 the behaviour observed for the GN and in general for the TKE closure are the same. Also, we stress once more that further halvening the mesh size was not possible for the GN. The finest computation we could perform until the final time is for $\Delta x=0.008$.

The behaviour observed allows to clearly demonstrate that the numerica dissipation has no impact on the computations performed with the TKE closure. This means that with this closure one could (or should) in principle use a non-dissipative numerical method to discretize the PDEs. The results, at least those for the GN equations, also show that the overall numerical dissipation when using the hybrid approach is larger on coarser meshes. 

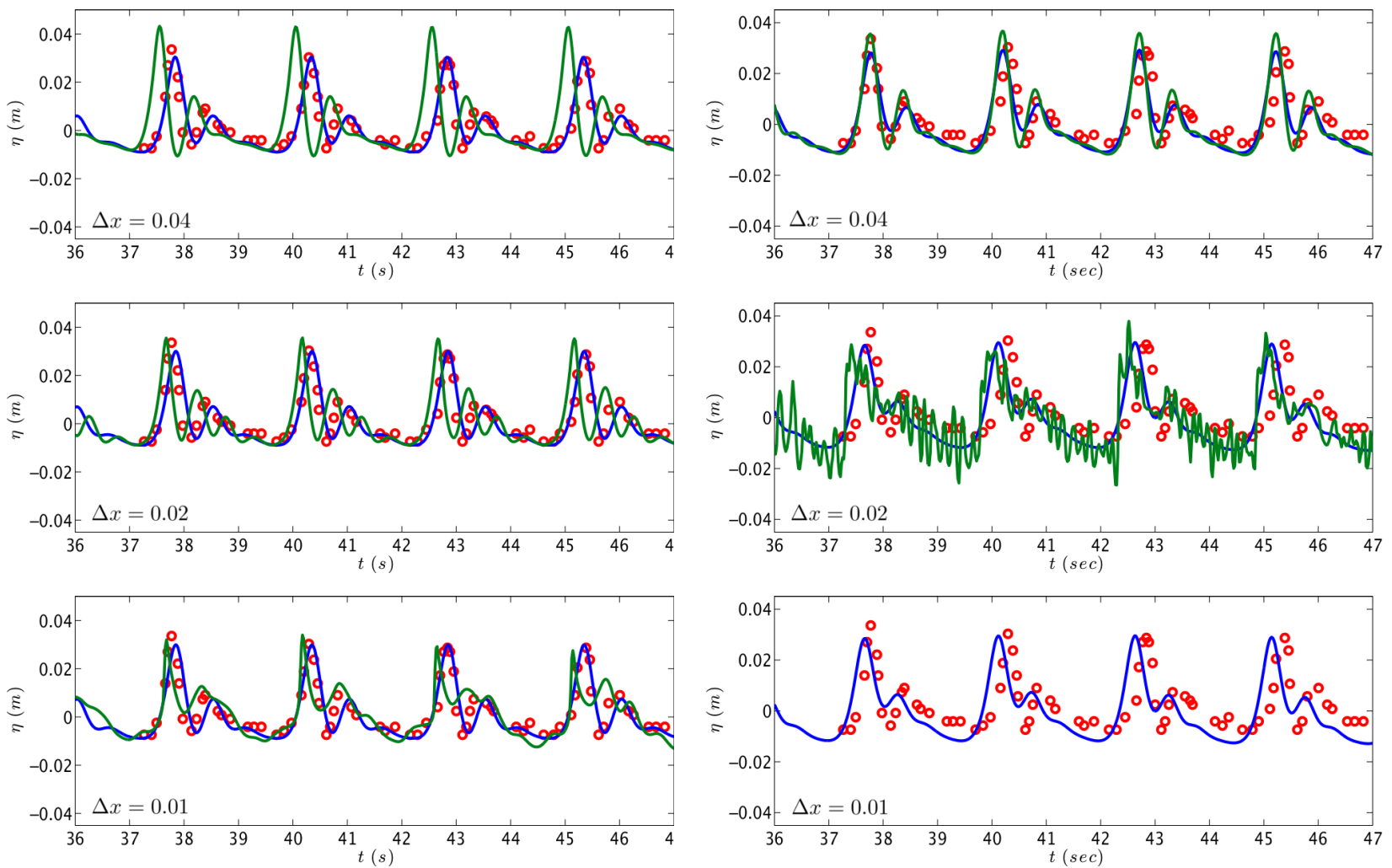

Figure 7: Time series of surface elevation at wave gauge 5 for the GN (left) and Nwogu (right) models using the TKE (blue) and Hybrid (green) wave breaking closure. Mesh size is $0.04,0.02,0.01 \mathrm{~m}$ from top to bottom.
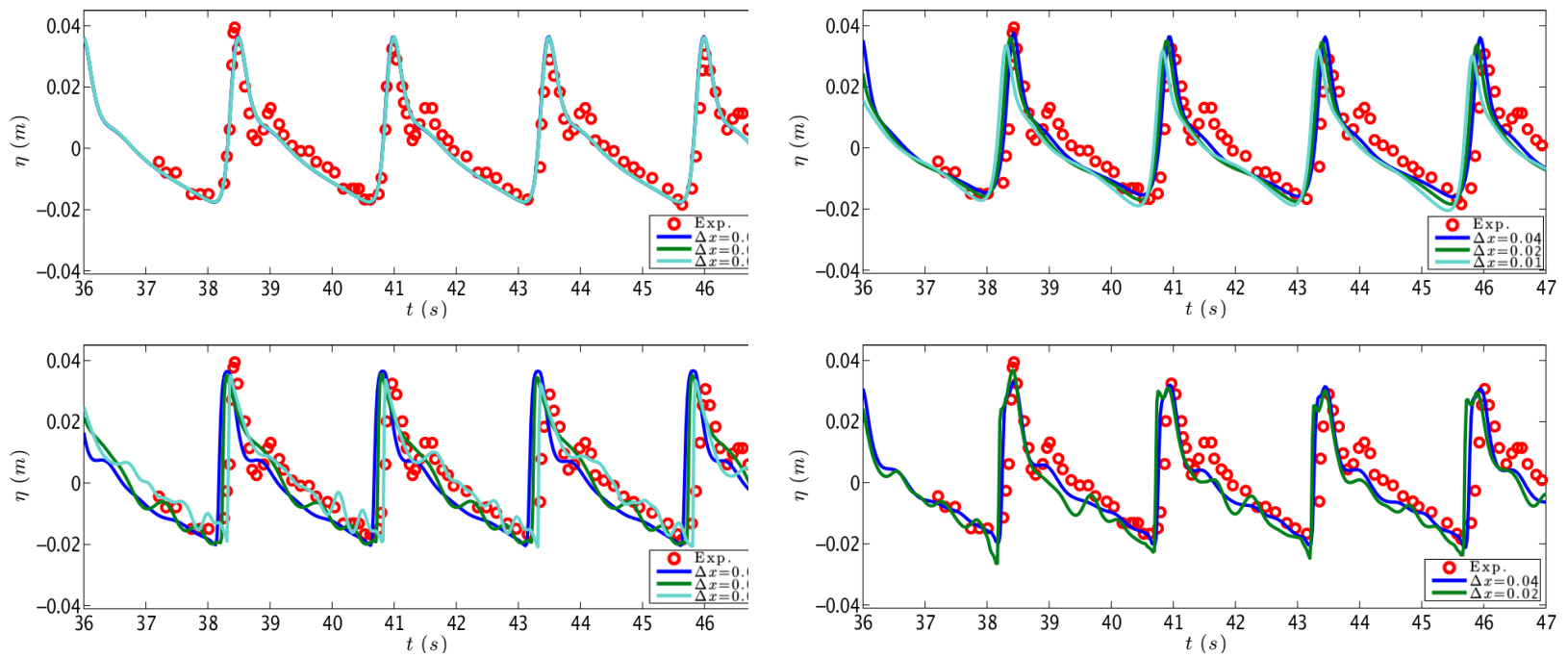

Figure 8: Time series of surface elevation at wave gauge 3: grid convergence for the GN (left) and Nwogu (right) models using the TKE (up) and Hybrid (down) wave breaking closure. Mesh size: 0.04m (blue), $0.02 \mathrm{~m}$ (green), $0.01 \mathrm{~m}$ (cyan). 

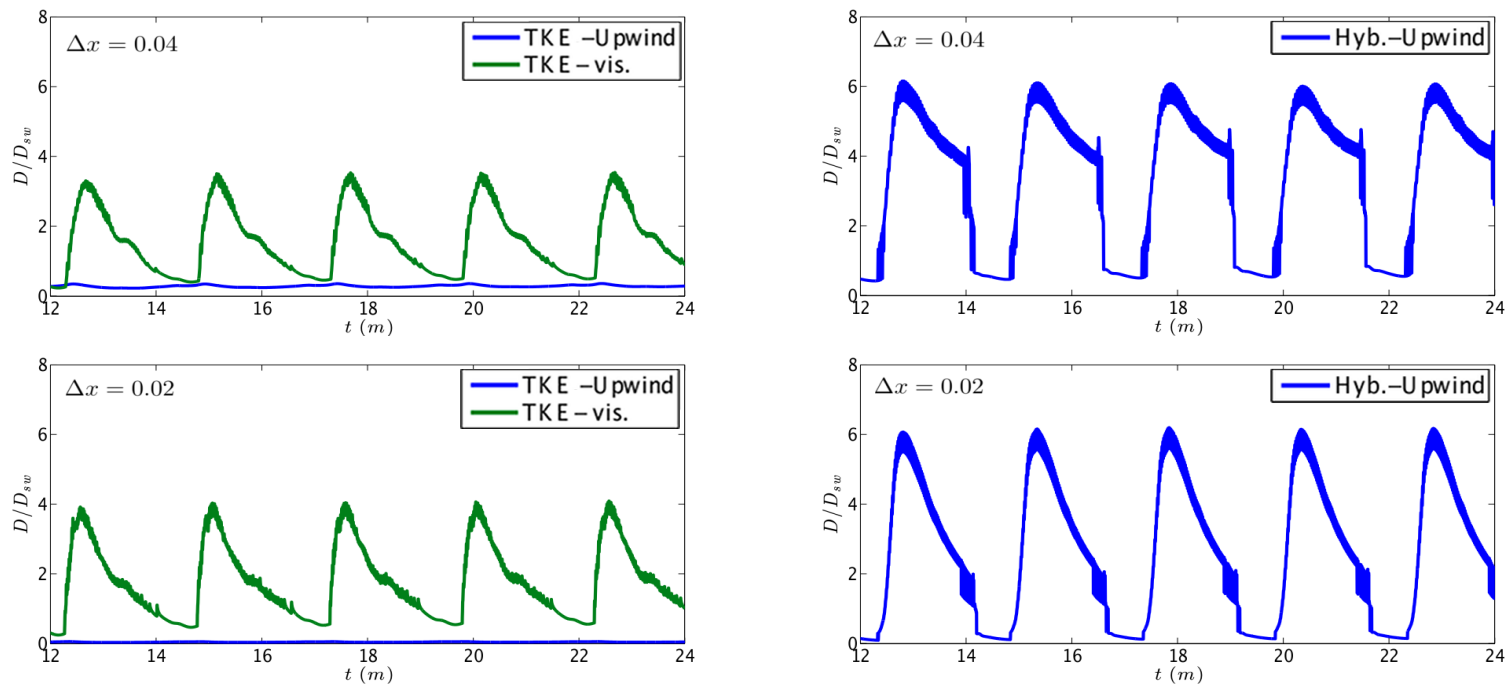

Figure 9: Energy dissipation profile for GN model using the TKE (left) and the Hybrid (right) closures for $\Delta x=0.04$ (top) and $\Delta x=0.02$ (bottom)

energy_bar_GI
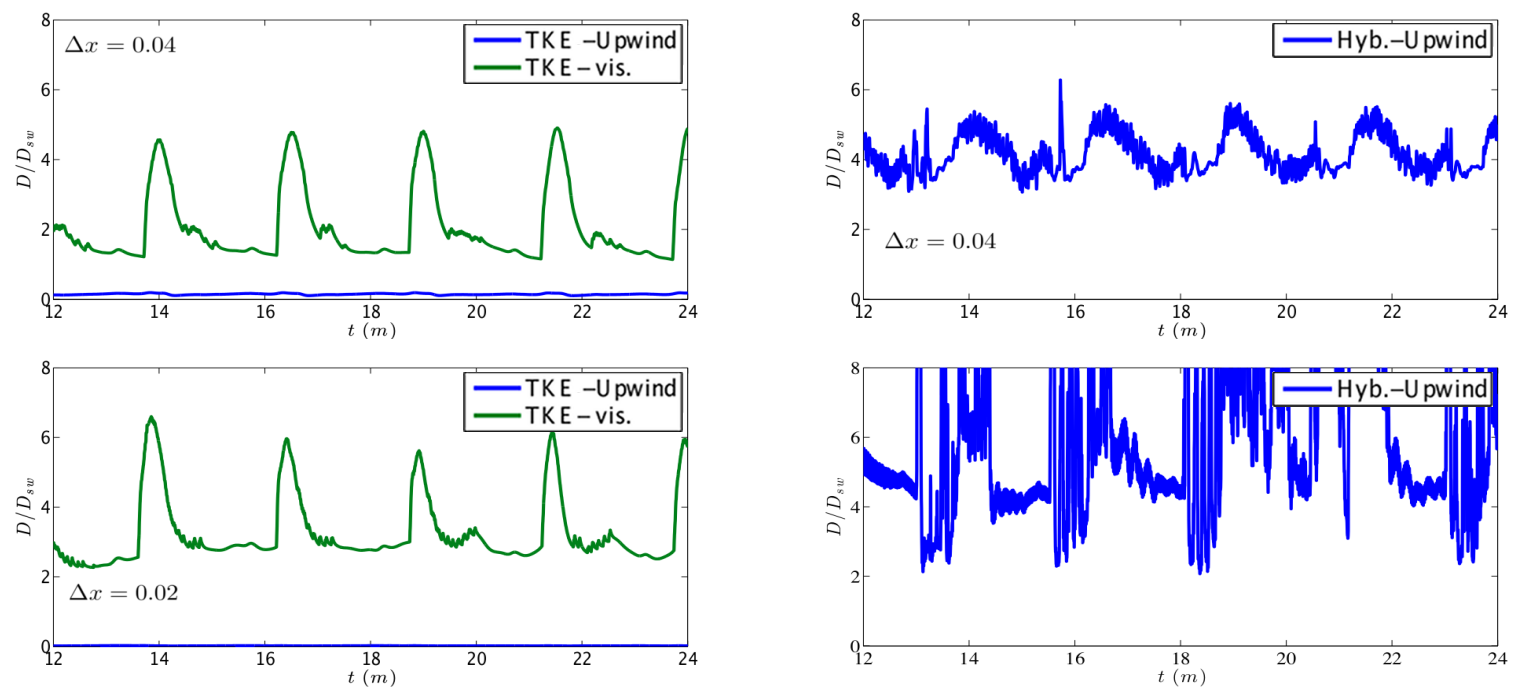

Figure 10: Energy dissipation profile for Nwogu model using the TKE (left) and the Hybrid (right) closures for $\Delta x=0.04$ (top) and $\Delta x=0.02$ (bottom)

\subsection{Solitary waves breaking on a slope}

One of the most intensively studied problems in long wave modelling is the solitary wave run-up on a plane beach, see for example $[12,16,18,47,75,95,96]$ among others. In this test case we want to study propagation, breaking and run-up of a solitary wave over a planar beach with a slope $1: 19.85$. With this famous test case we asses the ability of our model to describe shoreline motions and wave breaking when Synolakis [47] the wave breaks strongly both in the run-up and in the rundown phase of the motion. The GN and Nwogu's equations are tested and compared, using for each one the turbulent kinetic energy wave 
breaking model and the hybrid wave breaking model. The same holds for all the test cases that follows.

The computation domain is of $120 \mathrm{~m}$, where $x \in[-20,100]$. The $C F L$ used is 0.3 and sponge layer was applied off-shore with length $L s=5 \mathrm{~m}$. A Manning coefficient of $n_{m}=0.01$ was used to define the glass surface roughness used in the experiments. As before, computations have been run on three different meshes with size $\Delta x=[0.025,0.0125,0.0063 \mathrm{~m}]$. The parameters of the wave breaking criteria used in this test case are $\gamma=0.6$ and $\phi_{c}=30^{\circ}$ for both models. To properly capture the hydraulic jump generated at during backwash, the TKE parameters depend here both on the propagation model and on the type of breaking criterion satisfied. In particular, for unsteady waves the surface time variation criterion is the one activated. In this case we use $\kappa_{G N}=0.75, \sigma_{G N}=0.9$ for the GN model and $\kappa_{N}=0.8, \sigma_{N}=1.5$ for Nwogu's model. If the slope criterion is activated, we use instead higher values, namely we set $\kappa_{G N}=1.5$, $\sigma_{G N}=15.5$ and $\kappa_{N_{1}}=1.5, \sigma_{N}=1.5$.

Figure 11 compares the numerical surface profiles for the GN equations and the experimental measurements. The same is plotted for Nwogu's equations in figure 12 . The numerical solution was obtained using $\Delta x=0.05 \mathrm{~m}$. As expected, both mathematical models produced similar behaviour. Until time $t \sqrt{g / h}=10$ the solitary propagates to the shore and the two wave breaking models produce, as expected, identical results since wave breaking hasn't started yet. As expected the Nwogu's model gives a wave which overshoals and breaks slightly earlier compared to the one produced by the GN equations. The experimental wave breaks around $t \sqrt{g / h}=20$. The numerical solution for the hybrid model is represented like a bore storing the water spilled from the breaking wave behind the front. At time $t \sqrt{g / h}=20$ the turbulence model represents the solution as a triangular bore considerably closer to the experimental data than the hybrid one. Similar behaviour has been observed by other researchers that used eddy viscosity models [16-18]. At time $t \sqrt{g / h}=25$ the bore collapses at the shore, and both approaches show good qualitative agreement with the data. After that the wave starts to run-up, with a maximum run-up occurring at $t \sqrt{g / h}=45$. As the water recedes, a breaking wave is created near the still water level. The numerical solution is approximated as a hydraulic jump for both numerical models. It is fully resolved using both breaking models, since the breaking criterion recognises the hydraulic jump and the NSW equations are used for the hybrid model while the proper amount of viscosity is added by the turbulent kinetic energy model.

Figures 13 and 14 show the numerical results for both breaking phases (at time $t \sqrt{g / h}=20$ and $t \sqrt{g / h}=60$ respectively) while refining the mesh. Up to the authors knowledge it is the first time that such a study is performed for a (quasi-)steady hydraulic jump for an eddy viscosity type model. The first set of figures depict the breaking of the wave which travels on-shore for both GN (left column) and Nwogus equations (right column). We can clearly see the oscillatory nature of the hybrid wave breaking mechanism. The profiles obtained indicate some sort of convergence of the mean. However this is completely spoiled by the oscillations produced due to the switching between the two sets of equations. On the contrary the turbulent kinetic energy wave breaking mechanism remains stable and gives a convergent solution for both sets of equations. The second set of figures plot the same for the hydraulic jump formed at backwash. The difference between the two approaches is more accentuated here. It is quite hard to see a convergence for the hybrid results, while this is clearly the case for the TKE ones. We must mention that the GN equations combined with the hybrid model is blowing up after $t \sqrt{g / h}=60$ for $\Delta x=0.0063$, while Nwogu's equations are more sensitive to the hybrid formulation since numerical solution is obtained only for the fist two meshes.

We have repeated this test for a more non-linear initial wave with $\epsilon=0.5$, on the mesh with $\Delta x=$ $0.025 \mathrm{~m}$. The results obtained at incipient breaking before the runup and during backwash are reported on figure 15 . As before the hybrid mechanism produces oscillations, in both breakers, and it is very unstable for Nwogu model. Oscillations are clearly visible for the GN results with the hybrid breaking Smooth capturing of the breakers is obtained also in this case with the TKE model. Figures 16 ib and lit show again, 

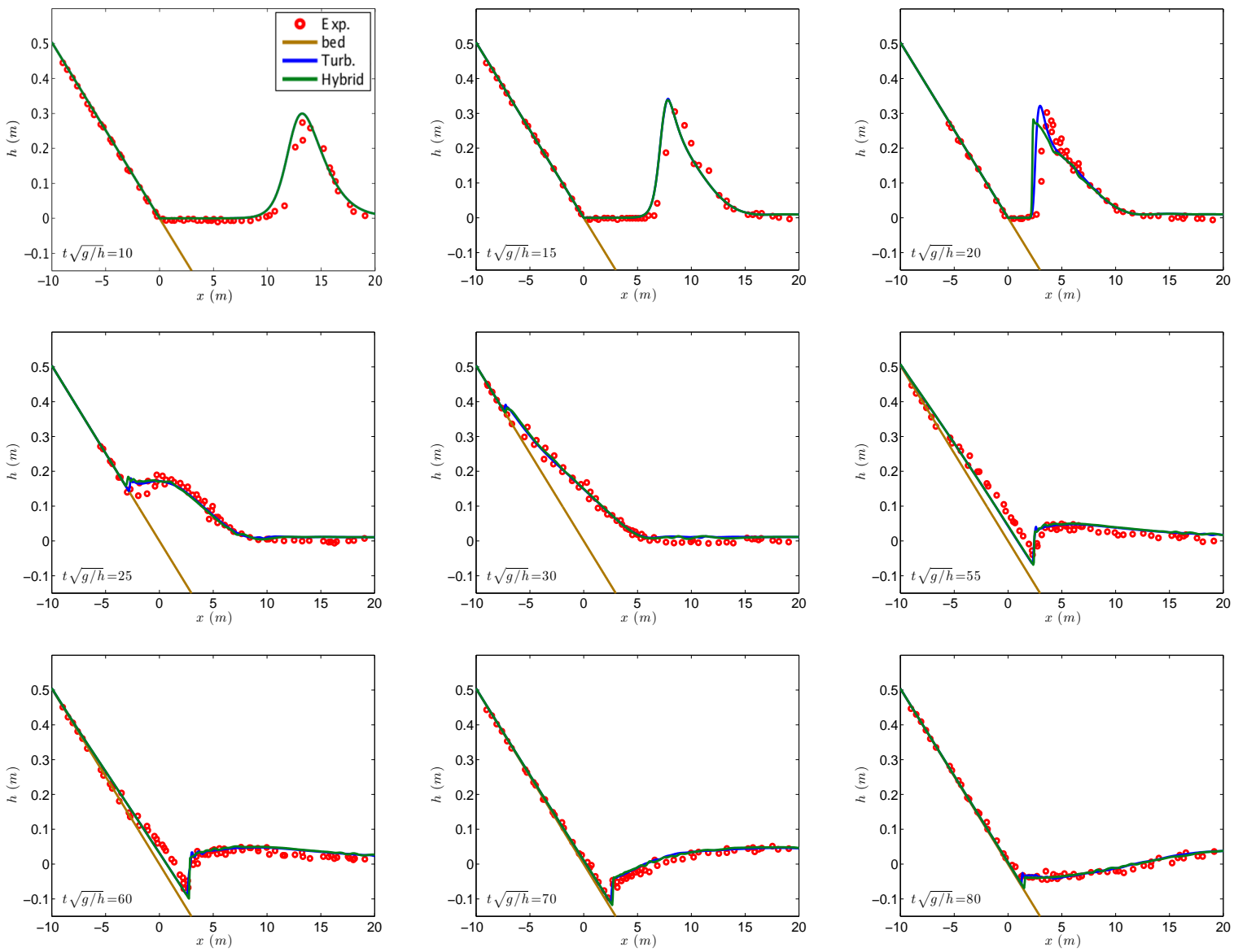

Figure 11: Free surface elevation of solitary wave run-up on a plane beach for the GN model.

the numerical results for both breaking phases while refining the meshes for the turbulent kinetic energy mechanism. The Hybrid closure is not converging since the oscillatory nature of the mechanism is more pronounced in this case.

\subsubsection{Dissipation mechanisms}

For both cases we now look at the contributions to the dissipation of energy. In this case, all three sources of dissipation are active (cf. section $\S \mathcal{5}$ ) : numerical dissipation $D_{\text {upwind }}$, dissipation due to friction $D_{\text {friction }}$, and the dissipation due to the eddy viscosity $D_{\text {vis }}$ when using the TKE closure. Let us first focus on the results for a nonlinearity of 0.28 reported in figures $18-19$ for the GN and Nwogu models. The results with the Nwogu model are on coarser meshes to allow some comparison on the behaviour of the hybrid closure on different meshes. The figures allow to see the dynamics of dissipation associated to the different phases of the flow. The fist breaking of the incoming wave is seen in all figures around time $5 s$, with the Nwogu model again showing earlier breaking certainly due to its over-shoaling characteristics. As the wave reaches higher bathymetries and the runup process starts, the friction takes over and dominates the flow, with no or very little contributions form the other terms. Dissipation is reduced to zero at the end of the runup, and if increases again during backwash, with again the friction dominating, and the other terms providing again 

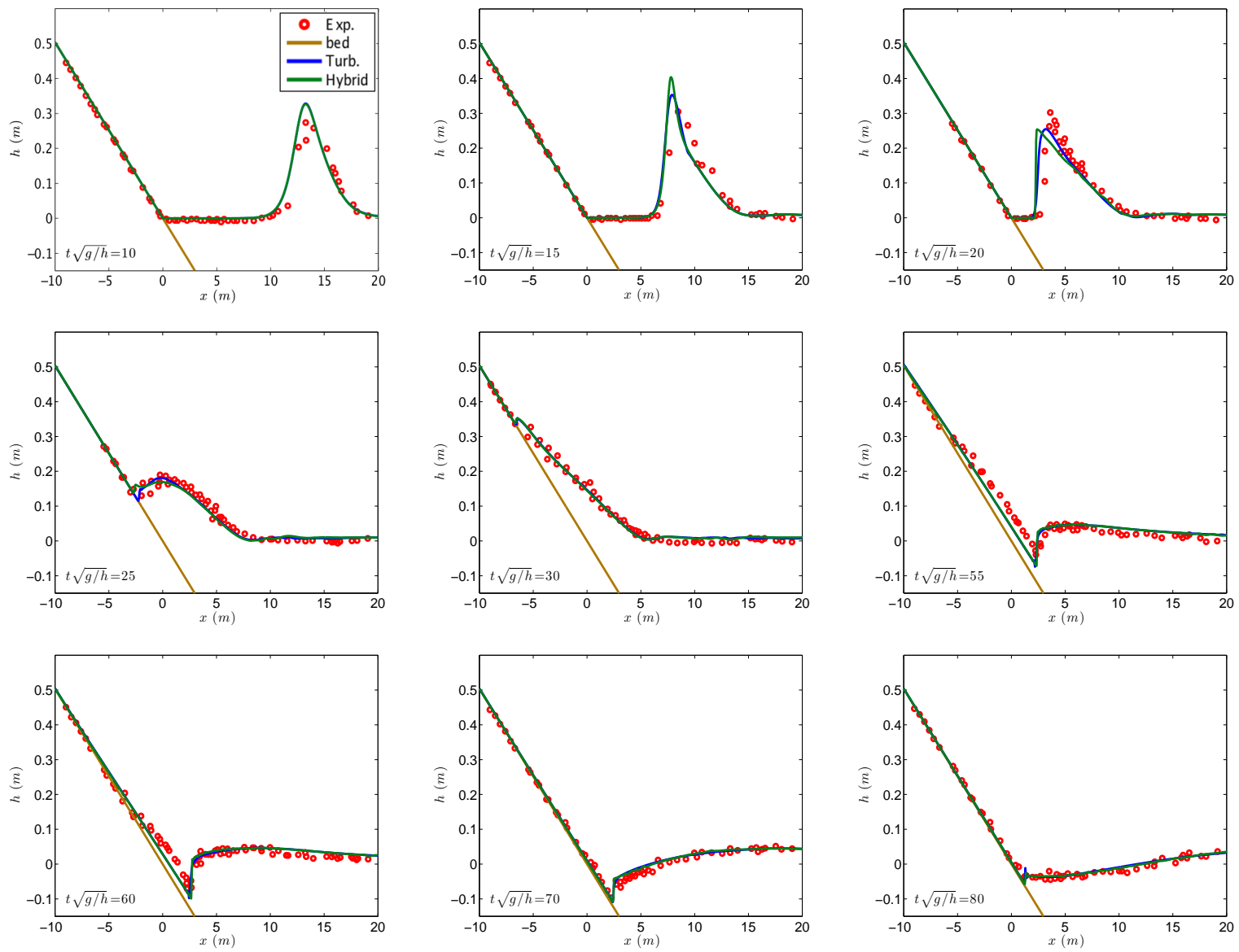

Figure 12: Free surface elevation of solitary wave run-up on a plane beach for Nwogu's model.

non negligible contributions around time $17 s-20 s$ when the hydraulic jump is formed. Note that these contributions arise from integrals in space. So the plot may lead to confusion as to which mechanism allows to capture the hydraulic jump. Indeed, the friction contributions are localized in the region very close to the wet/dry interface, and they would not allow to capture the hydraulic jump.

Looking at the behaviour of the different terms on the meshes considered, we can remark again that when using the TKE closure the numerical dissipation $D_{\text {upwind }}$ is not contributing, or providing very small contributions, throughout the flow. In he case of the hybrid closure, we can again see that it is indeed $D_{\text {upwind }}$ that provides dissipation during breaking. We can also see from figure 18 that this contribution is slightly larger on the coarser mesh, even though is is less clear that in the previous case. The oscillations observed during backwash in both the viscous contribution and numerical dissipation are associated to the intermittency of the breaking detection criterion. which is certainly something to be improved in the future. Finally, we remark that the behaviour for finer meshes is exactly the same, and that the finest meshes on which we managed to run this case until the final time with the hybrid closure are those mentioned earlier, namely $\Delta x=0.0063 m$ for the GN model, and $\Delta x=0.025$ for the Nwogu model.

We perform the same analysis for the case with a nonlinearity of 0.5 . The results are reported on figures 

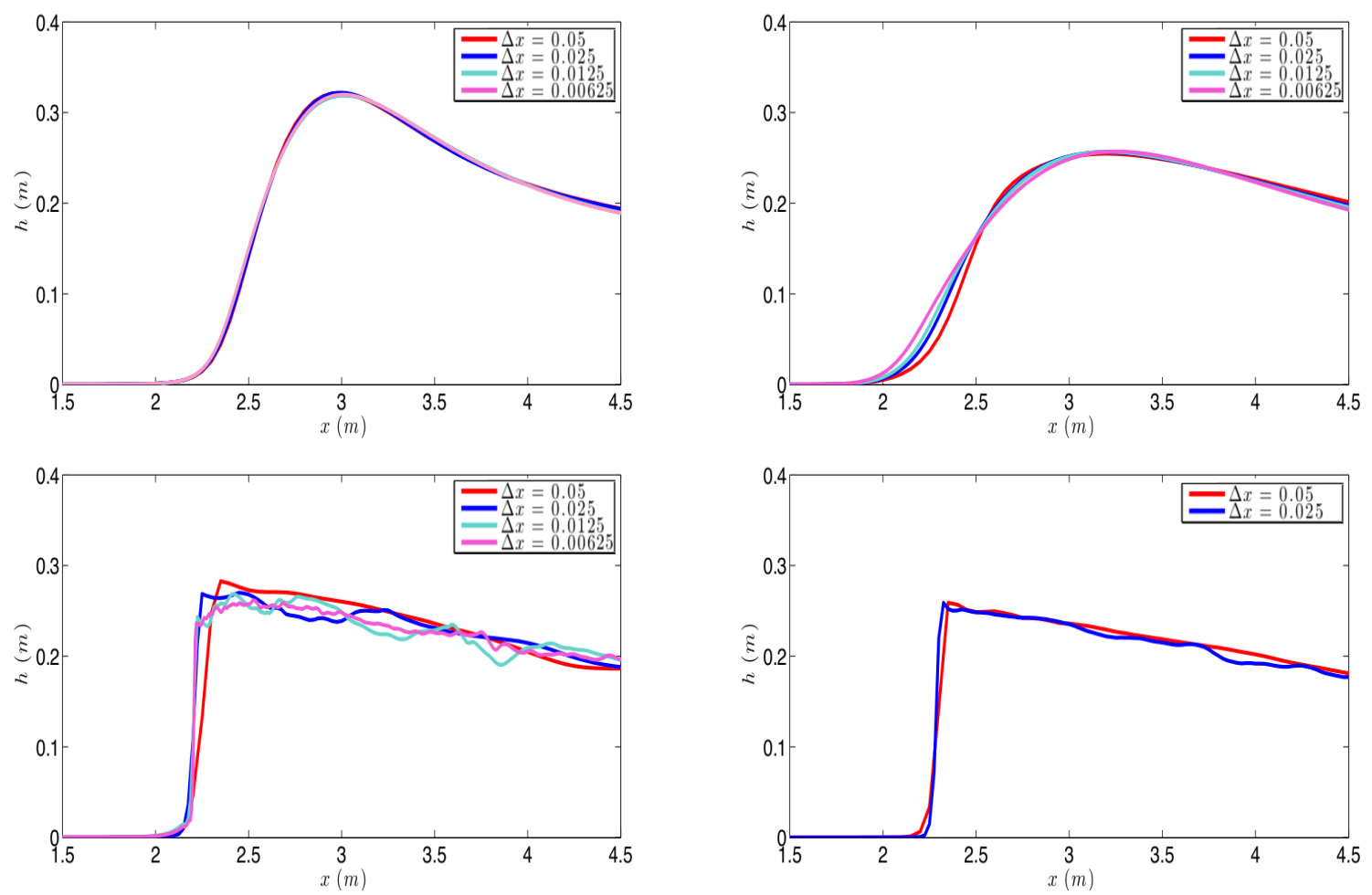

Figure 13: Breaking bore on different meshes for the GN (left) and Nwogu's (right) models, using the TKE (up) and the hybrid (down) wave breaking closure.

ene equersyyn QRey.0BD_Nwogu

20- 21. As mentioned already, in this case we could only run the Nwogu model with hybrid closure on the coarsest resolution of $\Delta x=0.1 \mathrm{~m}$. The dynamics observed in the figures are very similar to those of the previous case. Of course in this case the first breaking occurs earlier (around $2 s$ ) with the Nwogu model again providing an earlier breaking. We can again again see the friction dissipation taking over during the runup process, then decreasing, and increasing again during backwash. As before, breaking is re-activated to capture the hydraulic jump forming during backwash. We can again remark that when using the TKE closure the numerical dissipation is not contributing to the process, which is dominated by the terms embedded in the PDE. On the contrary, it is the numerical dissipation term that rules the dynamics of breaking. We can also see quite clearly that a considerable reduction of this contribution is obtained with mesh refinement. Again, the contributions of $D_{v i s}$ and of $D_{\text {upwind }}$ are quite oscilaltory during the backwash, and this is related to the intermittency of the detection mechanism. The meshes shown here are the finest we could run this case on until the final time with the hybrid closure.

As for the previous case, this analysis shows that when using the eddy viscosity closure the numerical dissipation plays very little or no role. This is motivation to look for non-dissipative/energy conserving schemes in this context. The mesh size seems to have an impact on the magnitude of the overall dissipation introduced during breaking. Finer meshes providing overall less dissipation. The TKE closure is very little sensitive to the mesh. This analysis also shows a very interesting interplay between the breaking and fiction dynamics. 

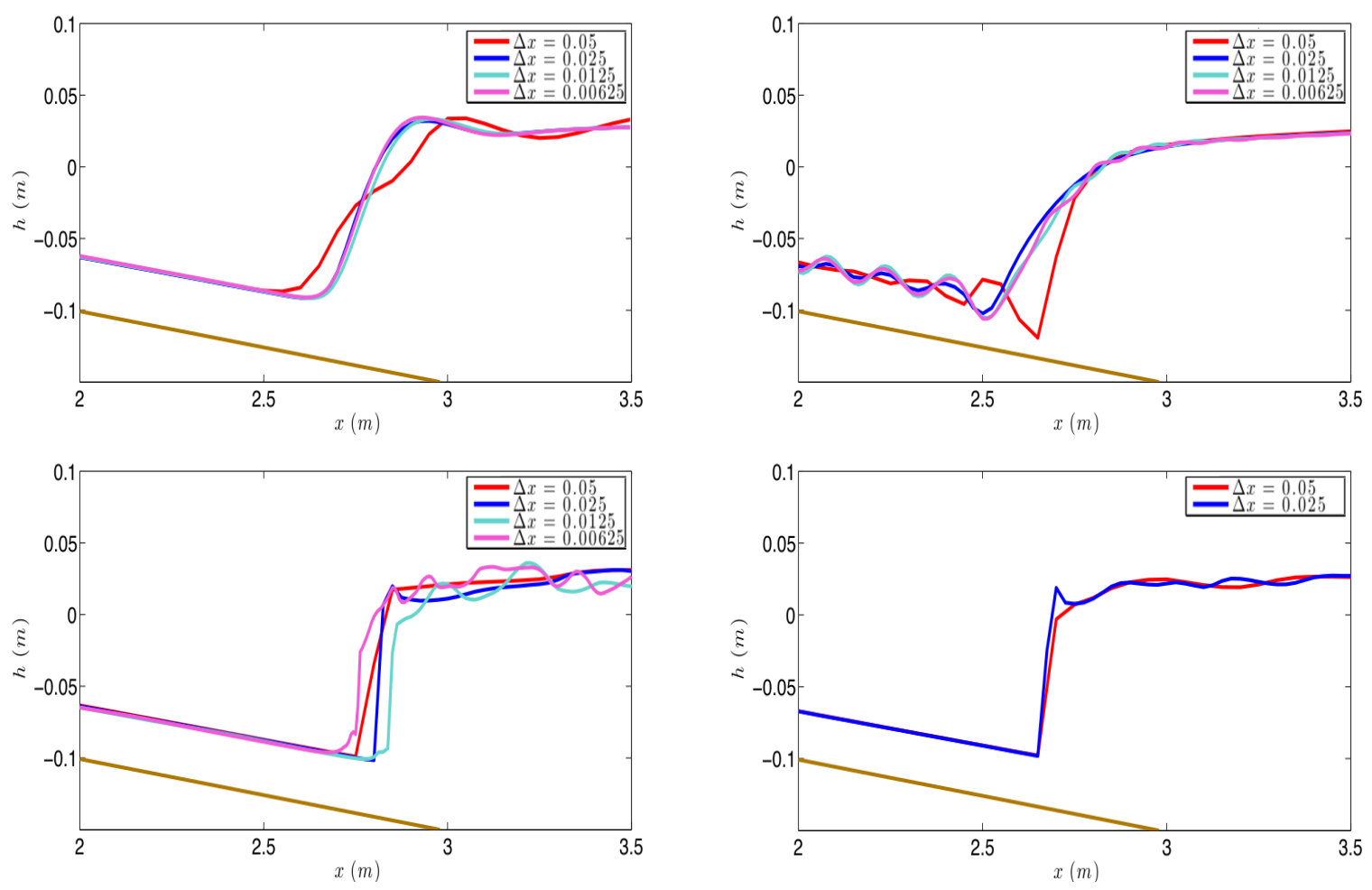

Figure 14: Hydraulic jump on different meshes for the GN (left) and Nwogu's (right) models, using the TKE (up) and the hybrid (down) wave breaking closure.

\subsection{Bore propagation and dissipation in function of the Froude number}

We consider in this test case the propagation of a breaking bore over a flat bottom. We have chosen this benchmark as its simple setting allows to perform some quantitative comparison between the discrete energy dissipation terms analyzed in the paper, and the exact theoretical shallow water dissipation, equation (14), for different values of the Froude number. The test is defined by an initial step which transforms to a bore. The initial solution is defined by

$$
\left\{\begin{array}{l}
h(x, 0)=\frac{1}{2}\left(d_{b}-d_{a}\right)\left(1-\tanh \frac{x}{a}\right)+d_{a} \\
u(x, 0)=\frac{1}{2}\left(u_{b}-u_{a}\right)\left(1-\tanh \frac{x}{a}\right)+u_{a},
\end{array}\right.
$$

where $d_{a}$ and $d_{b}$ are the water depth in front and behind the bore, $u_{a}$ and $u_{b}$ the corresponding depthaveraged velocities. In our case $u_{a}=0, d_{a}=1 \mathrm{~m}$ and $a=2 m$. For each Froude number $\left(F_{r}\right), u_{b}$ and $d_{b}$ are computed, solving the mass and momentum conservation conditions across the bore. For $F_{r}>1.4$ the initial step evolves into a breaking bore. More informations on the test case can be found in [97] and references therein.

The computational domain used is $x \in[-150,150], C F L=0.2$ and $\Delta x=0.1$. For this type of wave the parameter most sensitive to the onset of breaking is the time derivative of the elevation $\gamma$, which we have set here to $\gamma=0.4$. For the turbulence model we have used $\kappa_{G N}=\kappa_{N}=1.5, \sigma_{G N}=\sigma_{N}=0.8$ for the GN and Nwogu equations respectively. Figure 22 shows the propagated bore at $t=0,1,15 \mathrm{~s}$ for the 

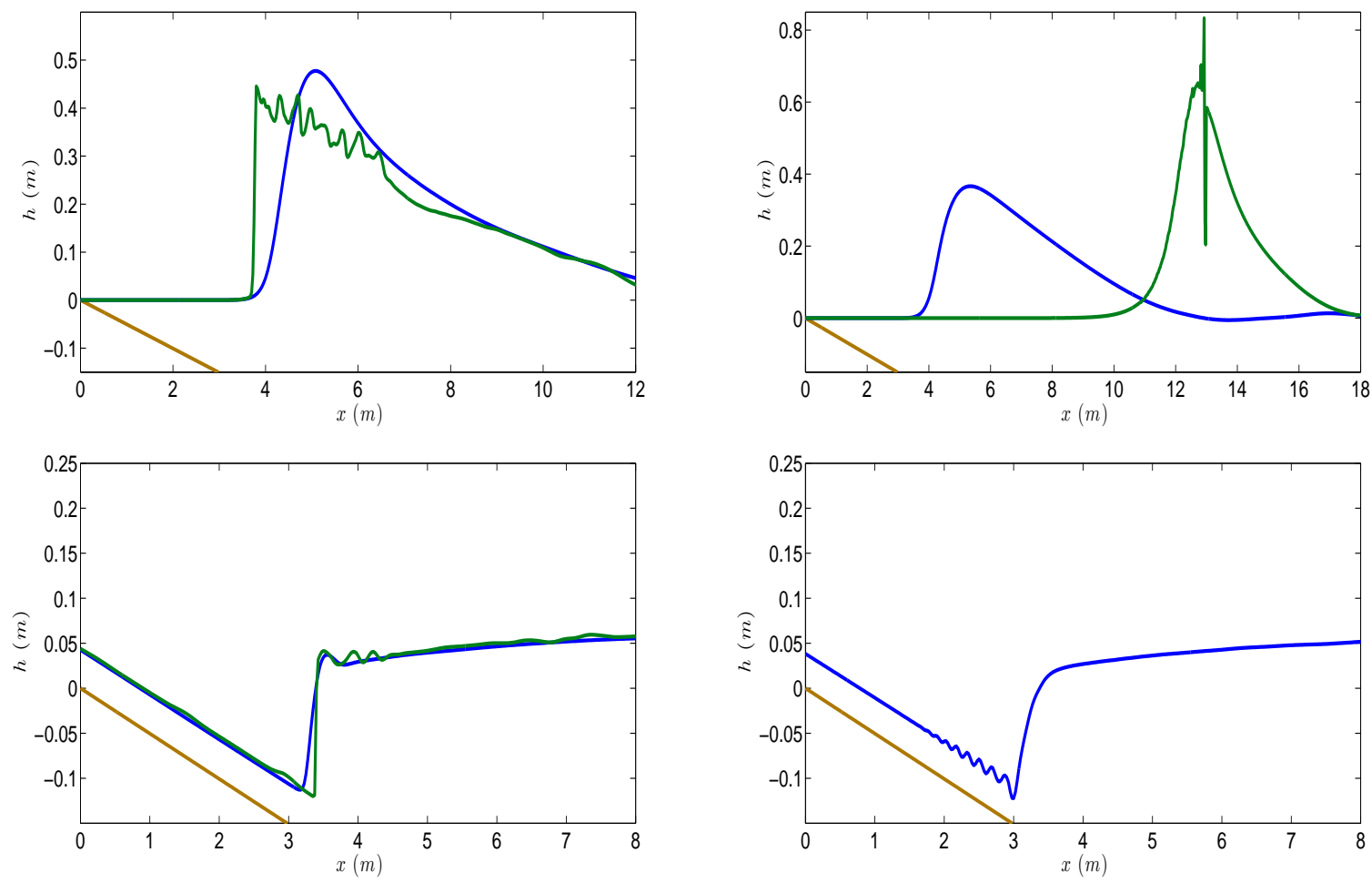

Figure 15: Breaking in the run-up (up) and the run-down (down) phase for GN (left) and Nwogu's equations (right) for $\epsilon=0.5$, using the TKE (blue) and the hybrid (green) wave breaking closure. Mesh size is $0.025 \mathrm{~m}$.
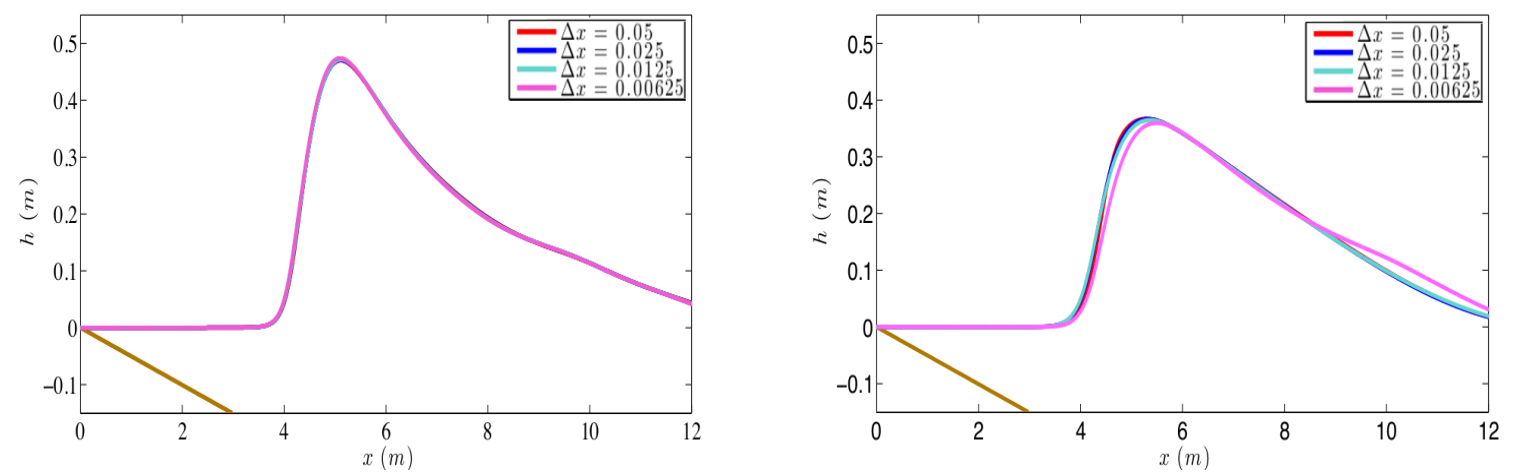

Figure 16: Breaking bore on different meshes for the GN (left) and Nwogu's (right) models for $\epsilon=0.5$, using the TKE wave breaking closure. Mesh size is $0.025 \mathrm{~m}$.

two models for a Froude number $F_{r}=2$. The bore is breaking as it propagates through the channel, and a slightly different behaviour is observed for the two breaking closures. Hybrid breaking provides a travelling shock, for both propagation models, while the turbulent closure presents a more diffusive behaviour, with a small overshoot before the bore for the GN model. 

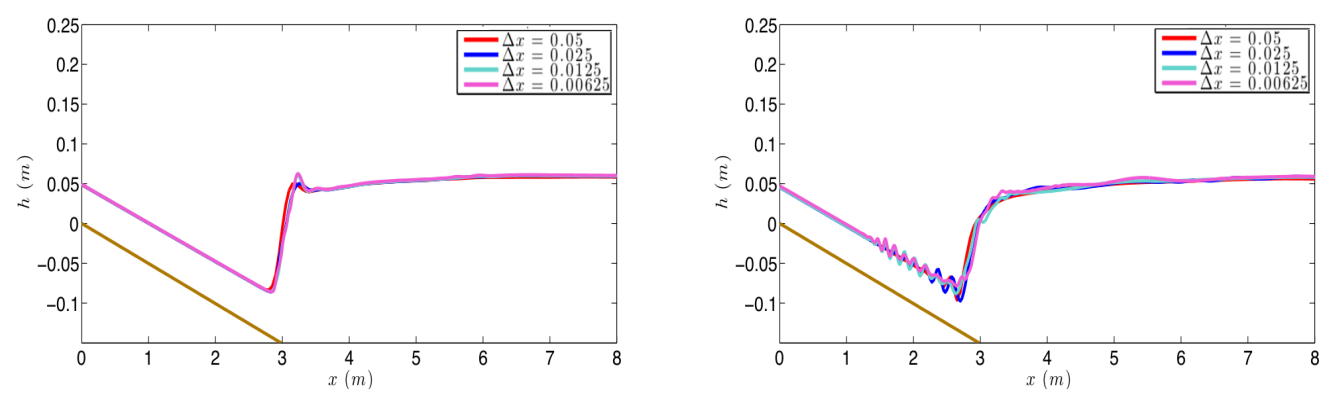

Figure 17: Hydraulic jump on different meshes for the GN (left) and Nwogu's (right) models for $\epsilon=0.5$, using the TKE wave breaking closure. Mesh size is $0.025 \mathrm{~m}$.

syn_con_0P5_1
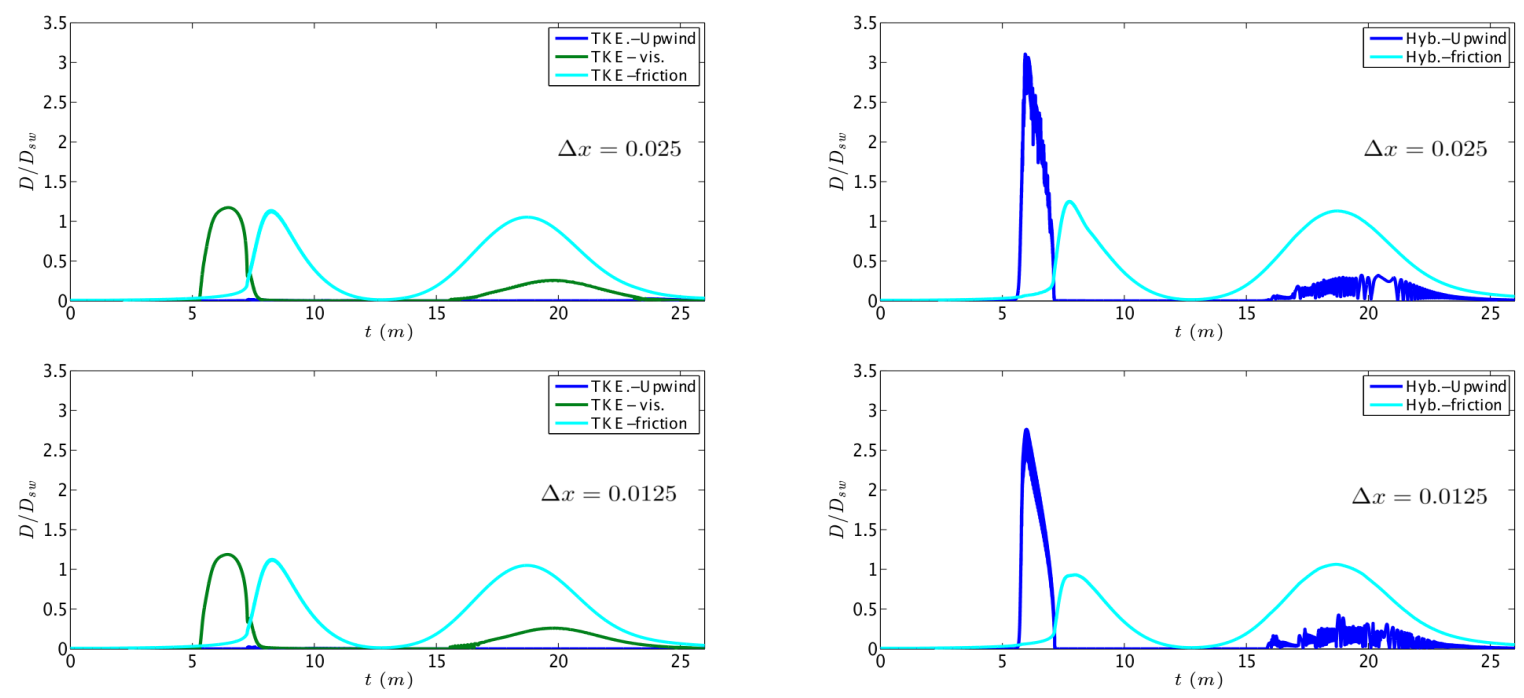

Figure 18: Energy dissipation profile for GN model using the TKE (left) and the Hybrid (right) closures on three different meshes: $\Delta x=0.025,0.0125 \mathrm{~m}$ from top to bottom. The nonlinearity of the wave is 0.28 .

The terms related to the upwind dissipation and to eddy viscosity evolve during the transformation of the solution into a bore, as shown on figure 22 , and quickly converge to a steady (in time) value, which is plotted in figures 23 and 24 against the shallow water dissipation (14), for different Froude numbers and on different meshes. Note that in this case, the wave breaking interface is located in correspondence of a constant solution region. This makes this case easier compared to the previous ones. This also reduces a lot the impact of mesh size on the final value of the dissipation, essentially dictated by the jump in water height. Nevertheless, exactly as the previous cases with the hybrid approach the initial development of the solution shows instabilities, for meshes finer that those reported in the figures, solution blow up. For the GN model, and for the range of Froude numbers tested, the TKE dissipation is within 10-15\% of the value predicted by (14), while the upwind terms basically provide a negligible contribution. Conversely, these terms are, when using the hybrid approach, within $6 \%$ of (14). As in the previous tests, this allows to demonstrate that the numerical dissipation does not contribute to the dynamics of wave breaking when using the TKE eddy viscosity closure. It also shows that the particular choice of eddy viscosity we performed allows to 

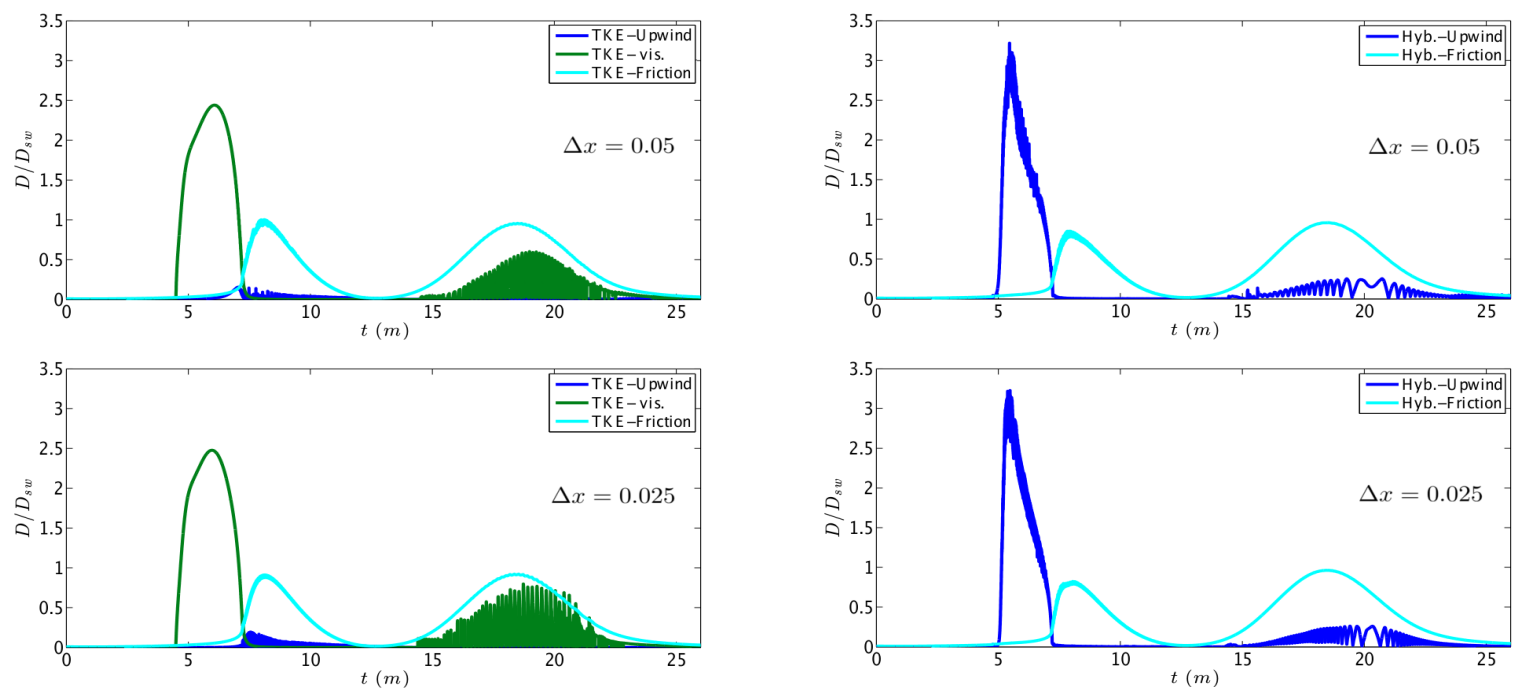

Figure 19: Energy dissipation profile for Nwogu model using the TKE (left) and the Hybrid (right) closures on three different meshes: $\Delta x=0.05,0.025 \mathrm{~m}$ from top to bottom. The nonlinearity of the wave is 0.28 .

energy_synop
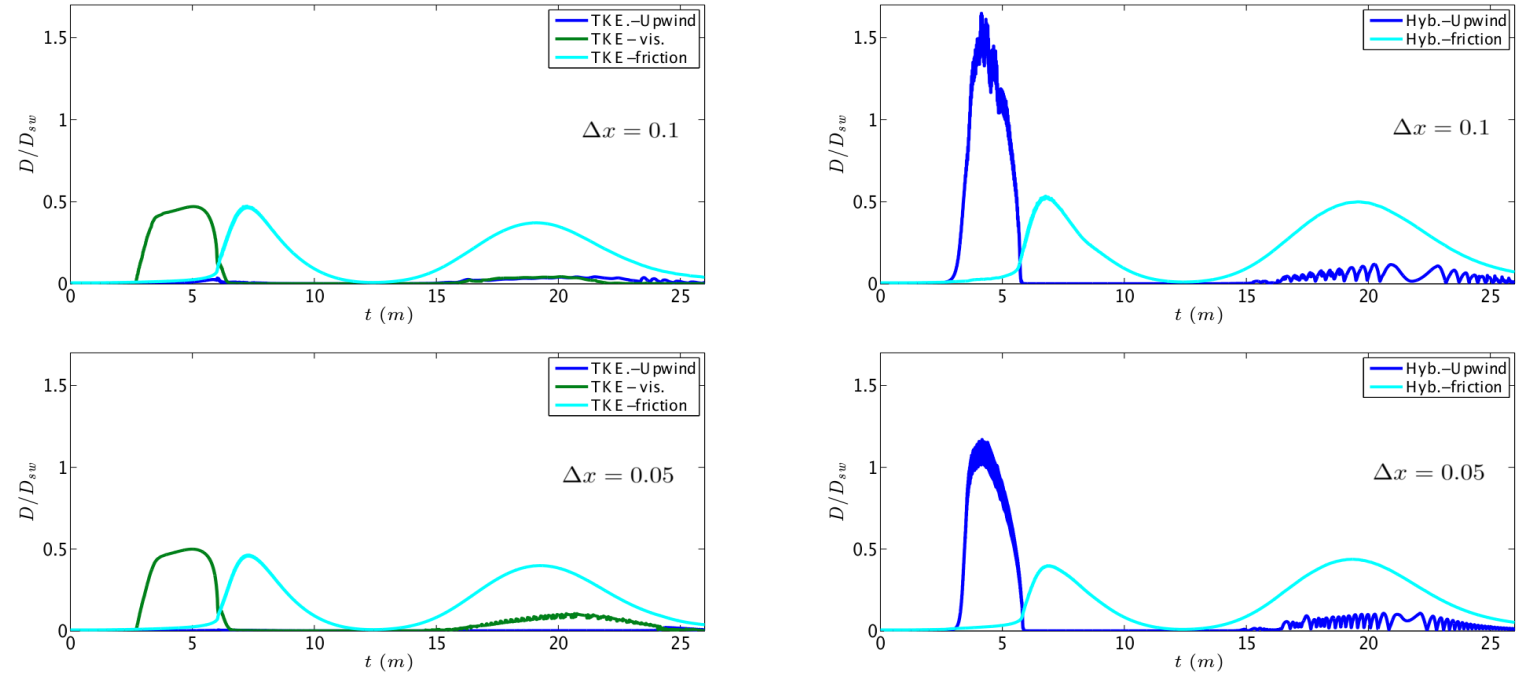

Figure 20: Energy dissipation profile for GN model using the TKE (left) and the Hybrid (right) closures on two different meshes: $\Delta x=0.1,0.05 \mathrm{~m}$ from top to bottom. The nonlinearity of the wave is 0.5 .

reproduce with some accuracy the behaviour with Froude number predicted by the classical formula (14). Similar conclusions can be drawn for the Nwogu model by looking at figure 24.

\subsection{Wave height and setup prediction}

The analysis of [74] shows that wave setup is very sensitive to the dissipation mechanism in wave breaking. So this is an interesting parameter to study for our purposes. To investigate this aspect we consider two of the experiments performed by Hansen and Svendsen [98]. These experimental studies consider several different regular waves shoaling and breaking on a sloping beach. Many authors have used these tests to 

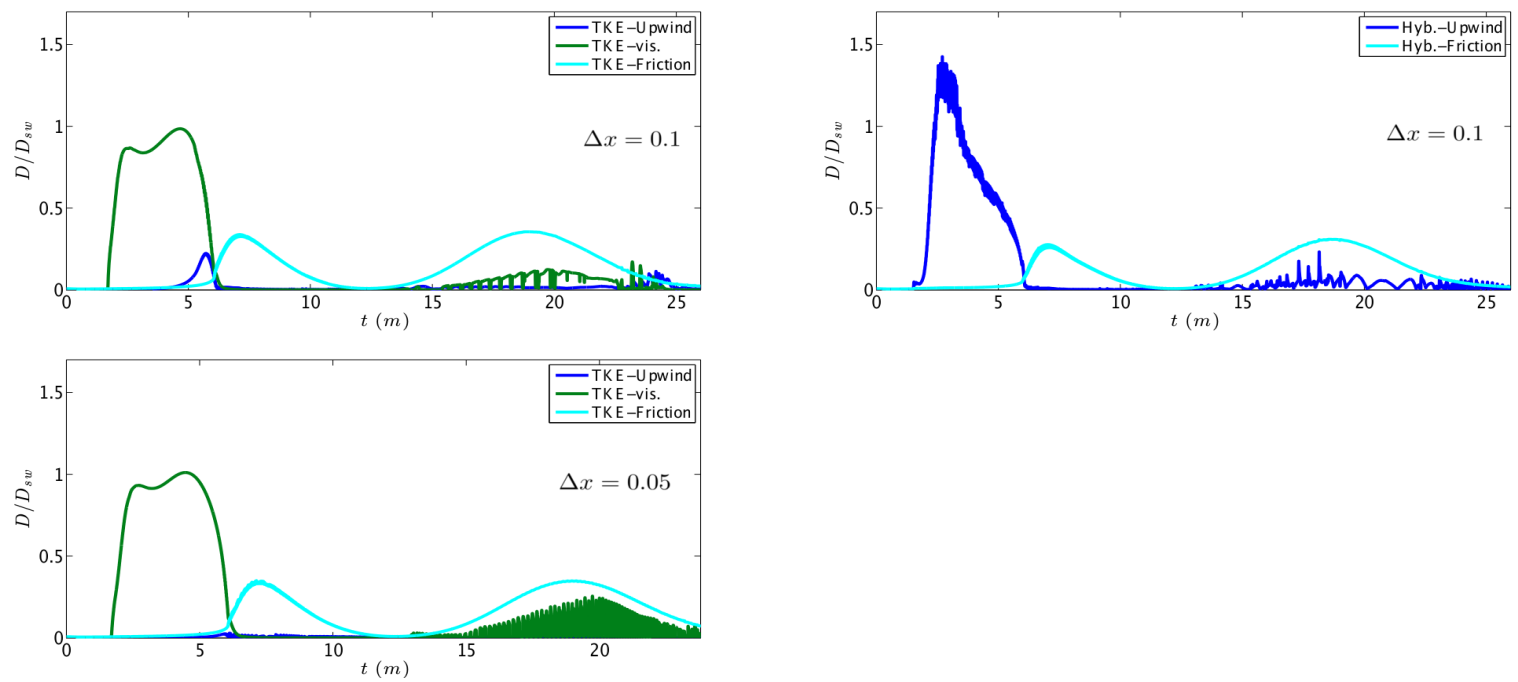

Figure 21: Energy dissipation profile for Nwogu model using the TKE (left) and the Hybrid (right) closures on three different meshes: $\Delta x=0.1,0.05 \mathrm{~m}$ from top to bottom. The nonlinearity of the wave is 0.5 .
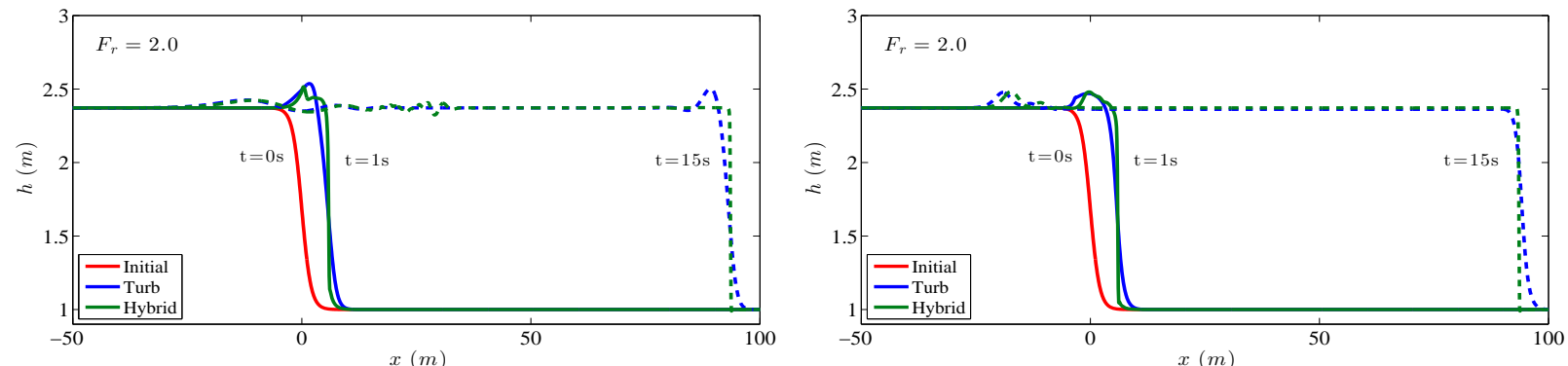

Figure 22: Free surface profiles at $t=0,1,15 s$ of hydraulic bores with $F r=2.0$. Left: GN model. Right: Nwogu model.
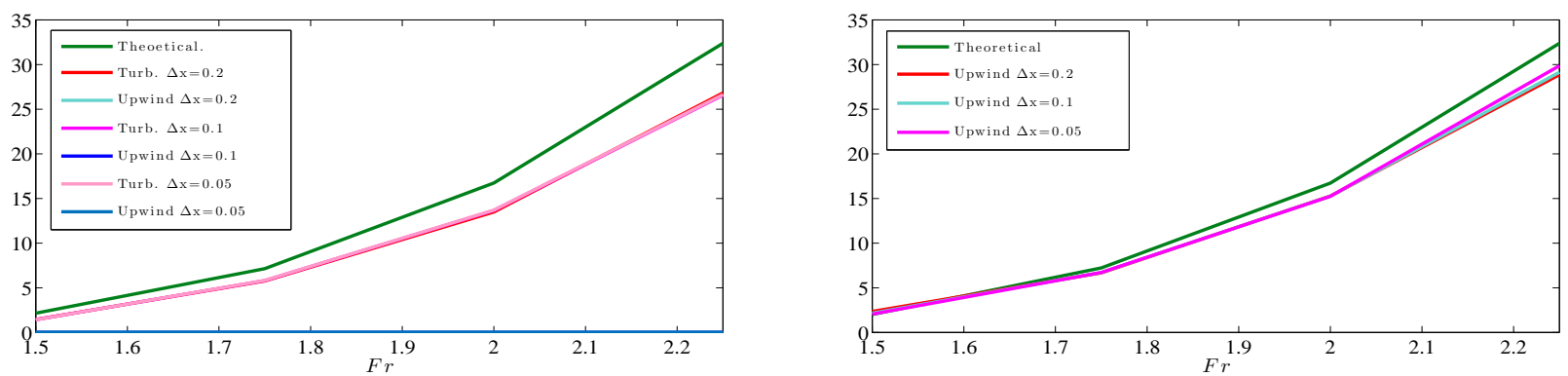

Figure 23: Energy dissipation profile for GN model using turbulent closure (left) and hybrid closure (right).

Gn_energy_1

validate their models and the associated breaking closures [6,12, 17, 23, 75].

We consider here two cases, one involving a spilling breaker, the second involving a plunging breaker. Regular waves are generated over a $0.36 \mathrm{~m}$ horizontal bottom, propagated shoaled and broke over a slope of $1: 32.26$. In the spilling breaking case (test number 05041) the regular wave's period $T$ is $2.0 s$, and 

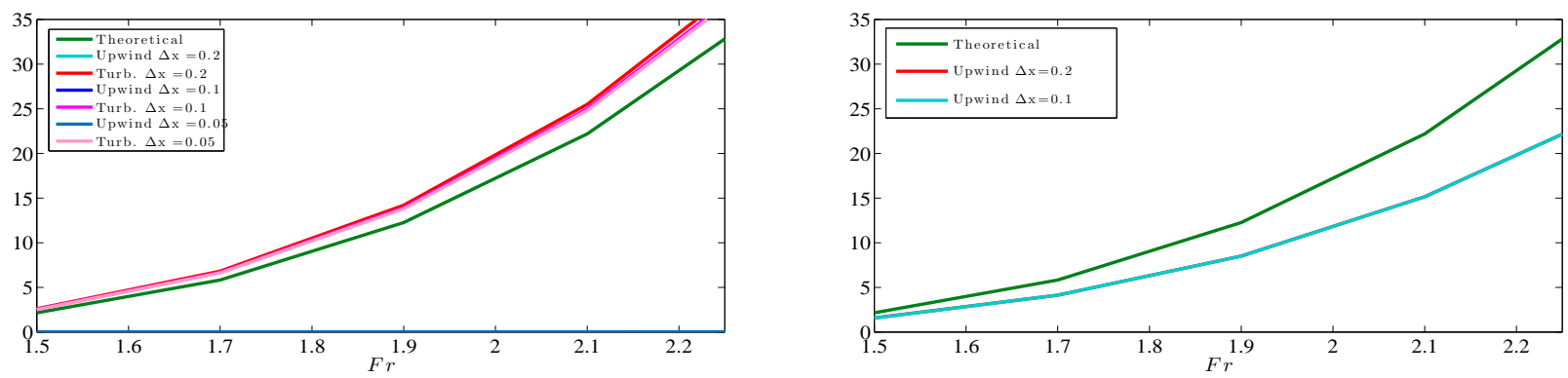

Figure 24: Energy dissipation profile for Nwogu model using turbulent closure (left) and hybrid closure (right).

the wave's height $H$ is $0.036 \mathrm{~m}$. The second test case (test number 03041) is a strong plunging breaking case with $T=3.33 \mathrm{~s}$ and $H=0.043 \mathrm{~m}$. The tests have been run on a $52 \mathrm{~m}$ long domain $x \in[-2626]$, discretised with cells of $\Delta x=0.02 m$, and with $C F L=0.3$. A sponge layer is applied offshore with length $L_{s}=5 \mathrm{~m}$. The wave making internal source was placed $14.78 \mathrm{~m}$ offshore from the toe of the beach, and bottom friction is neglected. The free surface elevation, recorded at wave gauges which placed every $0.1 \mathrm{~m}$., is analysed to compute the mean wave height, and the position of the mean water level (MWL). The value of $\gamma$ in the surface variation criterion equals to 0.5 for both models. Concerning the wave breaking closures, we have set $\kappa_{G N}=0.8, \sigma_{G N}=0.05$ and $\kappa_{N}=0.8, \sigma_{N}=0.4$, for the two GN and Nwogu models respectively.
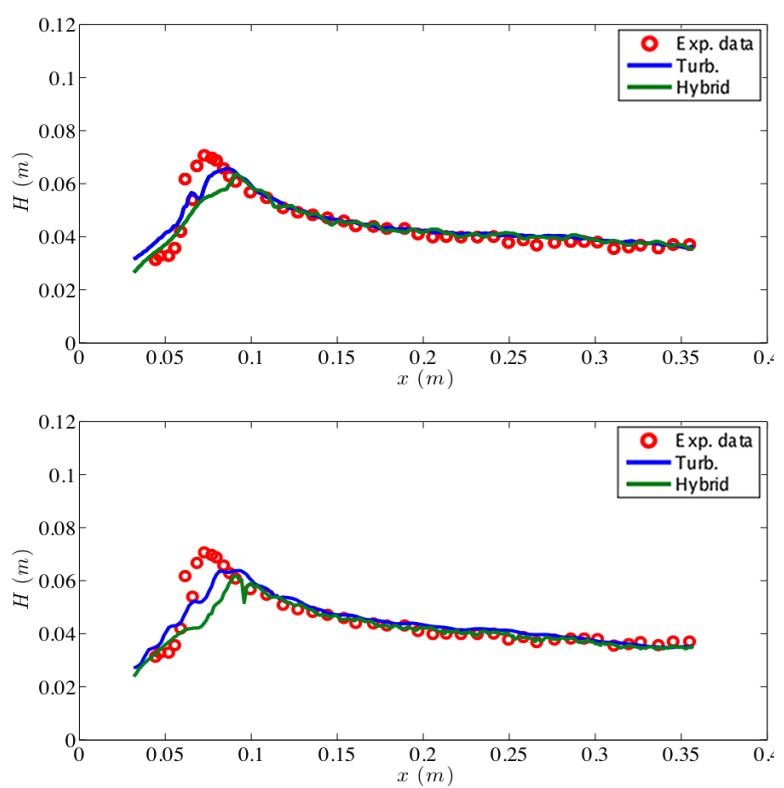
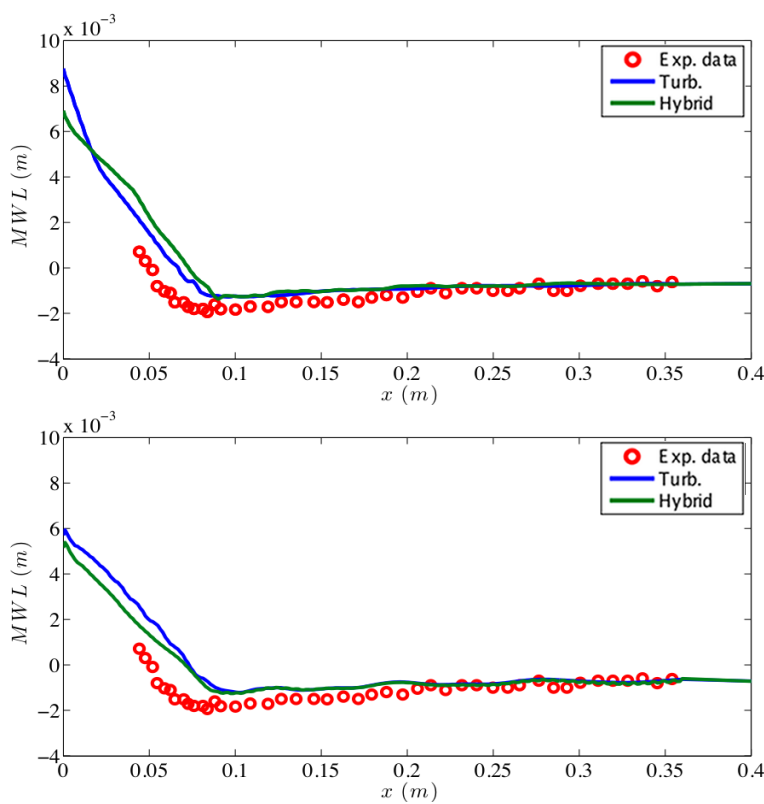

Figure 25: Computed and measured wave heights (left) and set-up (right) using equations. Test number 05041 (spilling breaking). Top: GN equations. Bottom: Nwogu equations. Blue line- TKE closure, green line- Hybrid closure.

The numerical results obtained for the two cases considered are reported on figures 25 and 26 , in terms of wave height (left) and mean water level (right). As before, the blue lines in the figures refer to the TKE results, while the green ones to the hybrid wave breaking, and the top row report the computations of the 

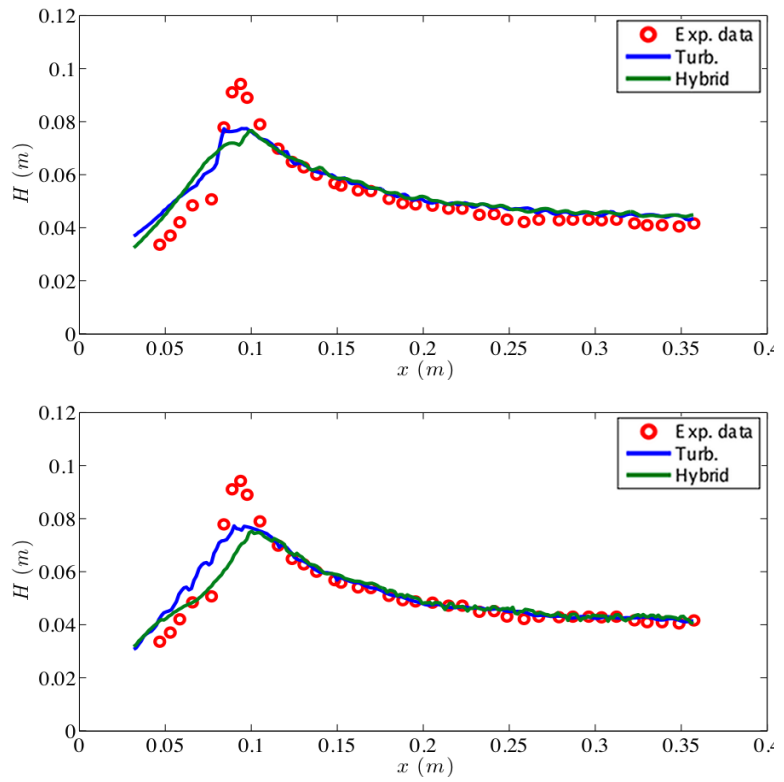
wave height and slope of the setup.
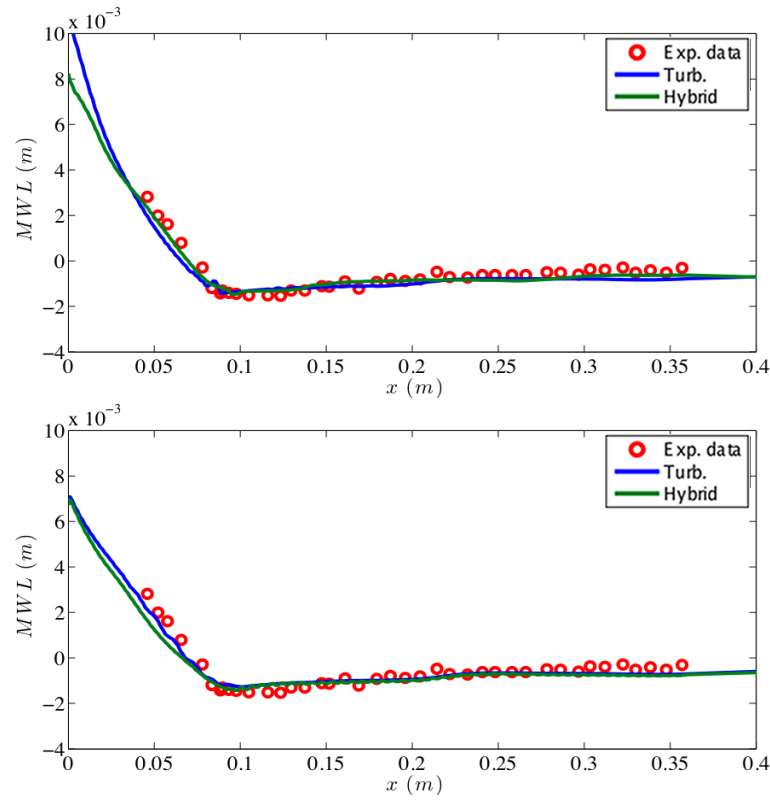

Figure 26: Computed and measured wave heights (left) and set-up (right) using equations. Test number 03041 (plunging breaking). Top: GN equations. Bottom: Nwogu equations. Blue line- TKE closure, green line- Hybrid closure.

GN model, while the bottom ones the results of the Nwogu equations.

For the spilling case, figure 25 seems to indicate that in all cases the detection criterion provides an early breaking. This of course alters the strength of the numerical breaking, which is less intense. This translates in a wave height decrease slower than the experimental one. Even so, the computations compare reasonably well with the experiments, especially when compared with results in the published literature $[6,12,17,23,75]$. This is confirmed by the mean water level plots. Although we can clearly observe the early start of setup, due to the early breaking, the slopes of the numerical signals are quite close to those of the experimental ones. According to the analysis of [74] this shows that the amount of dissipation introduced is correct. We stress that the differences between the TKE and hybrid approach are minor, even though we tend to consider the results obtained with the turbulence model slightly better in terms of both

\section{For the plunging case figure $\frac{\operatorname{case} 03041}{26, \text { the } 201}$} see that the breaking location is detected correctly in this case, even though both the GN and the Nwogu model provide an underestimation of the shoaling with both breaking closures. The wave height decrease is predicted with a slightly smaller slope, but the agreement with the data is quite satisfactory. The setup prediction is very good, with both location of the breaker and slope reproduced correctly by all models.

Some conclusions can be drawn from the implementation of this numerical test case. The first one is that both wave breaking closures allow to detect and handle both spilling and plunging breaking of regular waves. We stress that the parametrisation used for TKE closure is the same for the two cases considered. This shows the potential of this type of approach to provide a robust accurate energy dissipation rate, independently on the number of nodal points per wavelength, and on the nonlinearity of the problem. 


\subsection{Application: propagation, breaking, and overtopping of a 2D reef}

This next test case is reported as a complex application in order to show the potential of the modelling choices evaluated here to handle the interaction of the whole range of phenomena: dispersive propagation, shoaling, breaking, overtopping, reflection. The benchmark considered was initially proposed in [18,99], and later used by several authors for validation $[18,37,100,101]$. The problem involves a bathymetry consisting of a reef with a fore slope of $1 / 12$ and a crest of $0.2 \mathrm{~m}$ reef crest and an offshore water depth of $2.5 \mathrm{~m}$. The reef crest is exposed by $0.06 \mathrm{~m}$ and hides on the lee side a flat with a depth of $0.14 \mathrm{~m}$. Water height distributions at several time instants and water height time series in 14 wave gauges have been measured in the flume experiments at Oregon State University within the PhD work of V. Roeber [99] (see also [18]). A sketch of the reef geometry, showing the positioning of the wave gauges, is reported in figure 27 . The initial state consists of a solitary wave of amplitude $a=0.75 \mathrm{~m}$ which propagates onshore, shoals and breaks in front of the reef crest. Walls are present at both ends of the domain. We refer to $[18,99]$ for a more detailed description of the experimental and computational setup. Our results have been computed on a mesh with size $\Delta x=0.05$, and setting $C F L=0.3$. Manning friction has been used, with a Manning coefficient $n_{m}=0.012$. Both wave breaking detection criteria are used with $\gamma=0.6$ and $\phi_{c}=30^{\circ}$. Concerning the TKE closure $\kappa_{G N}=0.75, \sigma_{G N}=0.8$ and $\kappa_{N}=1.2, \sigma_{N}=1.5$ but when a hydraulic jump is detected the values are set to $\kappa_{G N}=1.5, \sigma_{G N}=15.5$ and $\kappa_{N}=3.5, \sigma_{N}=16$.

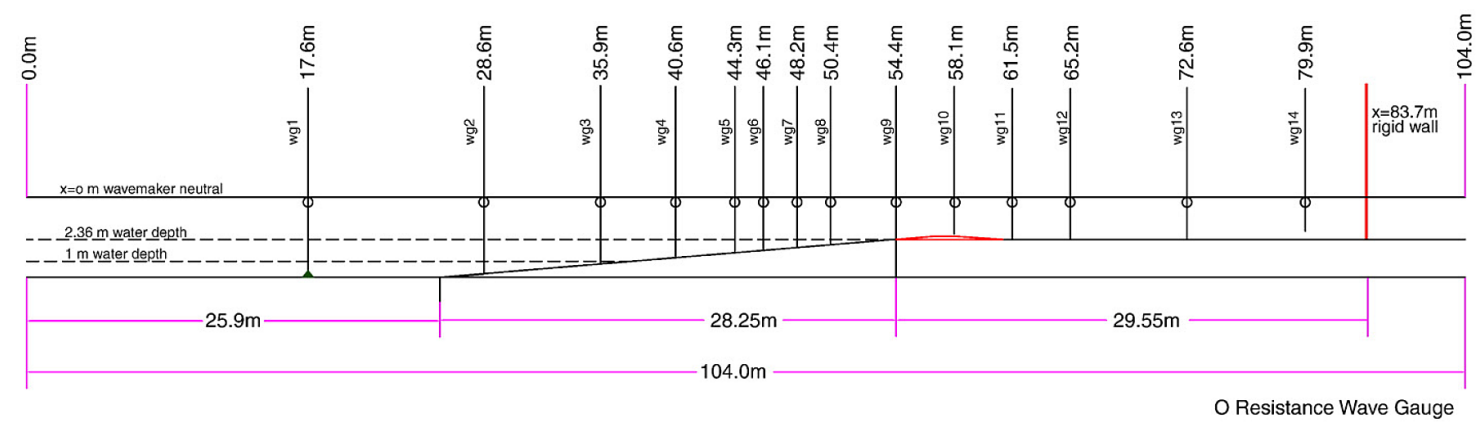

Figure 27: 2D reef geometry and wave gauge locations. Adapted from [18].

To visualise the results we group snapshots of the free surface in three phases : propagation and shoaling of the initial soliton (figure 28$) ; 0$ vertopping and formation, propagation and reflection of a bores on the lee

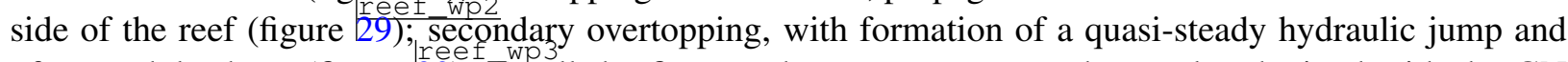
of an undular bore (figure $\frac{\mathrm{r}}{30}$ ). . In all the figures, the top rows report the results obtained with the GN model, the bottom rows refer to the results of the Nwogu model, the blue lines are those obtained with the TKE breaking model, and the green lines are those of the hybrid breaking treatment. Symbols refer to the experimental values provided in [99].

The figures show that all models allow, on this mesh resolution, a quite satisfactory prediction of the water height. The differences between different choices appear to be minor. We can mention that, at least in our implementation both the fully and the weakly nonlinear models tend to predict the moving bores on the lee side with some phase advance. This, at least in our implementation, is more pronounced for the fully nonlinear GN mode, as we can see e.g. on figure 29 (central and right column). This behaviour is independent on the breaking closure adopted. We can also remark that when using the hybrid wave breaking with the Nwogu equations some over-prediction of the amplitude of the undulating bores is observed.

To have some more insight in the capabilities of the models, we analyze the water height time series 
in gauges WG5, WG9, WG10, and WG12. The plots are reported on figures $\frac{\mid r e e f}{31}$ and $^{2} 32$. propagation of the waves is visible in WG5 and, at for the fore side undulating bores, in WG9. We can see that all the models capture correctly the shoaling of the initial solitary, and that despite a visible phase lag, provide a quite reasonable amplitude and frequency of the undulating bores on the fore side, as it can be seen e.g. in the WG5 series on figure $\frac{r}{31}$, for times larger than $70 s$, and in WG9 after $80 s$. In WG5 we can see again the over-ampification of the amplitude of the undular bores for the Nwogu model with hybrid wave breaking.

Concerning breaking, we can see the first breaker approximation very well reproduced from the WG9 series at time around $34.5 \mathrm{~s}$. The hydraulic jump forming at $55 \mathrm{~s}$ is also well reproduced in amplitude, albeit with a phase advance. Similar observations can be made when looking at figure $\frac{\text { reet }}{32}$. The WG12 results, in particular, show an excellent agreement for the first four bores. All the models give an under-prediction of the water level behind the slowly moving hydraulic jump which forms behind the main right-going bore (time 38s). The first reflected bore at time roughly $50 \mathrm{~s}$, as well as the second hydraulic jump forming after the second overtopping (time $60 \mathrm{~s}$ ) are also very well captured by the models. The later reflections present instead a visible phase error, albeit correct in amplitude. Lastly, the WG10 results in the same figure show a nice capturing of the first two overtopping phases, although an over-prediction of the water height is also observed. The later overtoppings are affected by a phase advance already mentioned for the bores responsible for them.

Overall we consider the results quite good for all the models. Some of the differences w.r.t. the experimental water heights we are convinced that are also due to the definition of this quantity in presence of air entrainment at the free surface, as it was the case for the experimental breakers. We stress very strongly that with the current implementation the simple TKE breaking closure can handle without any problem simultaneous breakers of different types, and of different intensities. For this test, as for all the others analyzed in the paper, the fully nonlinear GN model with TKE closure provides the most robust combination.
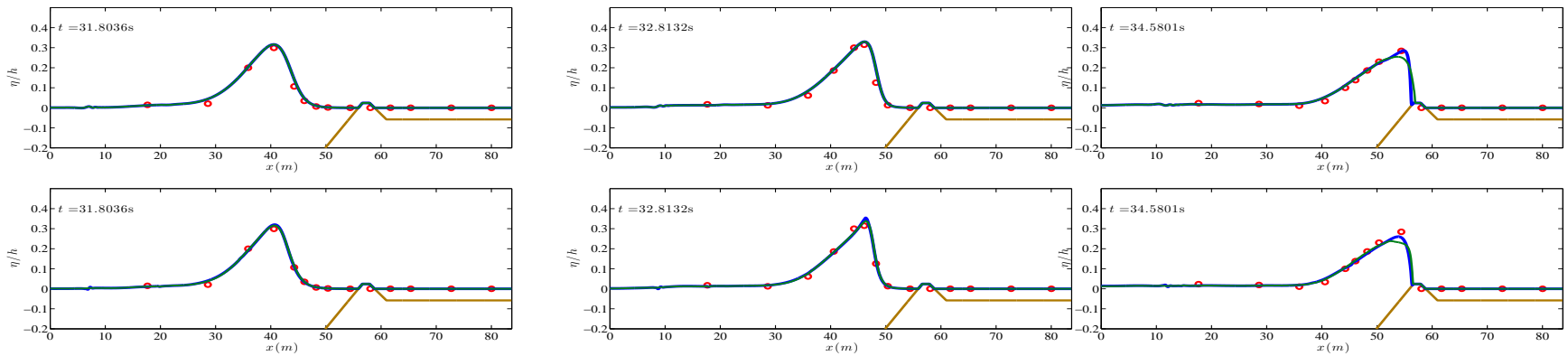

Figure 28: Overtopping of a 2D reef. Propagation, shoaling, and overtopping phases.Top row: GN model. Bottom row: Nwogu model. Blue lines: TKE breaking closure. Green Lines: hybrid wave breaking closure. Left: $t=31.8036 s$. Middle: $t=32.8132 s$. Right: $t=34.5801 s$.

\section{Conclusions}

We have considered the issue of wave breaking closure when using weakly dispersive Boussinesq propagation models. We studied weakly and fully nonlinear models representative of classical and well known models/codes such as BOUSS-2D [3,4], Funwave [5, 6], Coulwave [7, 8], BOSZ [9], MIKE21 [10], TUCWave $[11,12]$, and others. We have in particular focused on the enhanced equations of Nwogu [49], and on a 

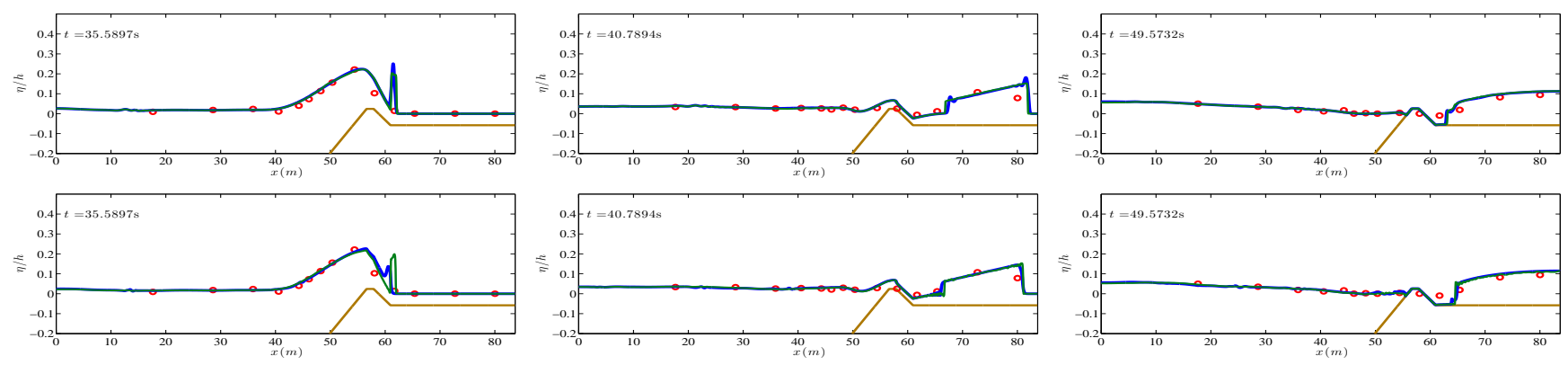

Figure 29: Overtopping of a 2D reef. Bore formation and propagation behind the reef. Top row: GN model. Bottom row: Nwogu model. Blue lines: TKE breaking closure. Green Lines: hybrid wave breaking closure. Left: $t=35.5897 \mathrm{~s}$. Middle: $t=40.7894 \mathrm{~s}$. Right: $t=49.5732 \mathrm{~s}$.
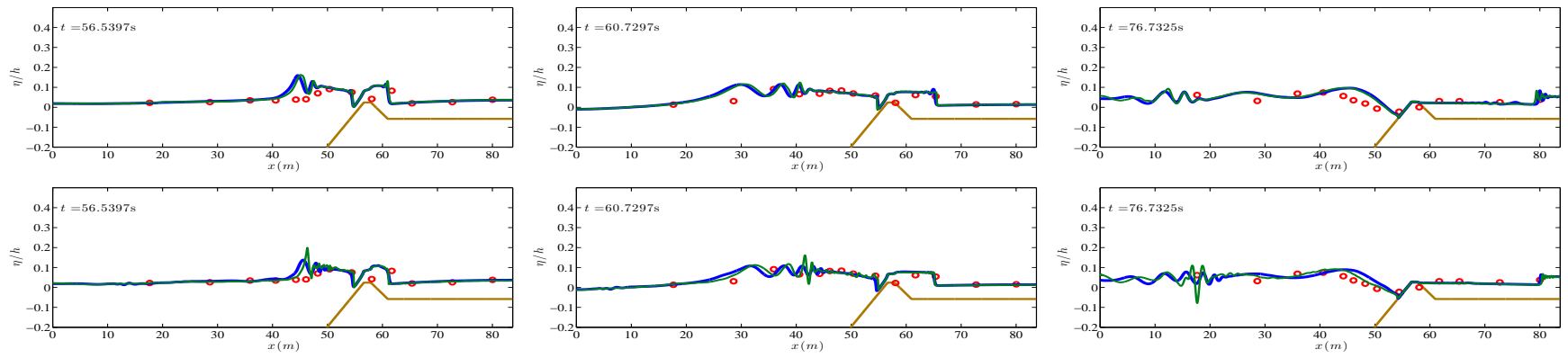

Figure 30: Overtopping of a 2D reef. Second overtopping and undular bore formation. Top row: GN model. Bottom row: Nwogu model. Blue lines: TKE breaking closure. Green Lines: hybrid wave breaking closure. Left: $t=56.5397 \mathrm{~s}$. Middle: $t=60.7297 \mathrm{~s}$. Right: $t=76.7325$.

frequency enhanced version of the Green-Naghdi system in the form proposed in [37,52]. We have compared the now popular hybrid closure initially proposed in [36], with an eddy viscosity closure based on an adaptation of the turbulent kinetic energy closure model of [1], modified to be consistent with the detection mechanisms proposed of [12,37], and also used here. The study performed has involved: a systematic analysis of the behaviour of the two closures for different mesh sizes; the use of dissipation monitors, consistent with the available theory of entropy dissipation for conservation laws [69,72], to study the dynamics of breaking for several cases; thorough evidence of the equivalent capabilities of the two approaches to provide satisfactory results.

Our results indicate that indeed, at least with the (rather standard) implementation proposed here, both closure approaches allow to describe correctly wave transformation and breaking at large scales. We have shown that when using the TKE eddy viscosity closure the numerical dissipation plays a negligible role, which motivates to look for non-dissipative/energy conserving numerical methods in the future. Also, the results clearly show the reduced sensitivity to the mesh of this approach compared to the hybrid one. The analysis of the wave breaking of solitary waves on a slope also has allowed to quantitatively study the interplay of the dissipation introduced by friction, eddy viscosity, and numerical dissipation.

Of course, one has to keep in mind that the computational cost required by the TKE closure is higher then the one of the hybrid closure. We judge this overhead justified by the increased robustness.

This preliminary study would benefit from further investigation using both improved numerics (e.g. en- 

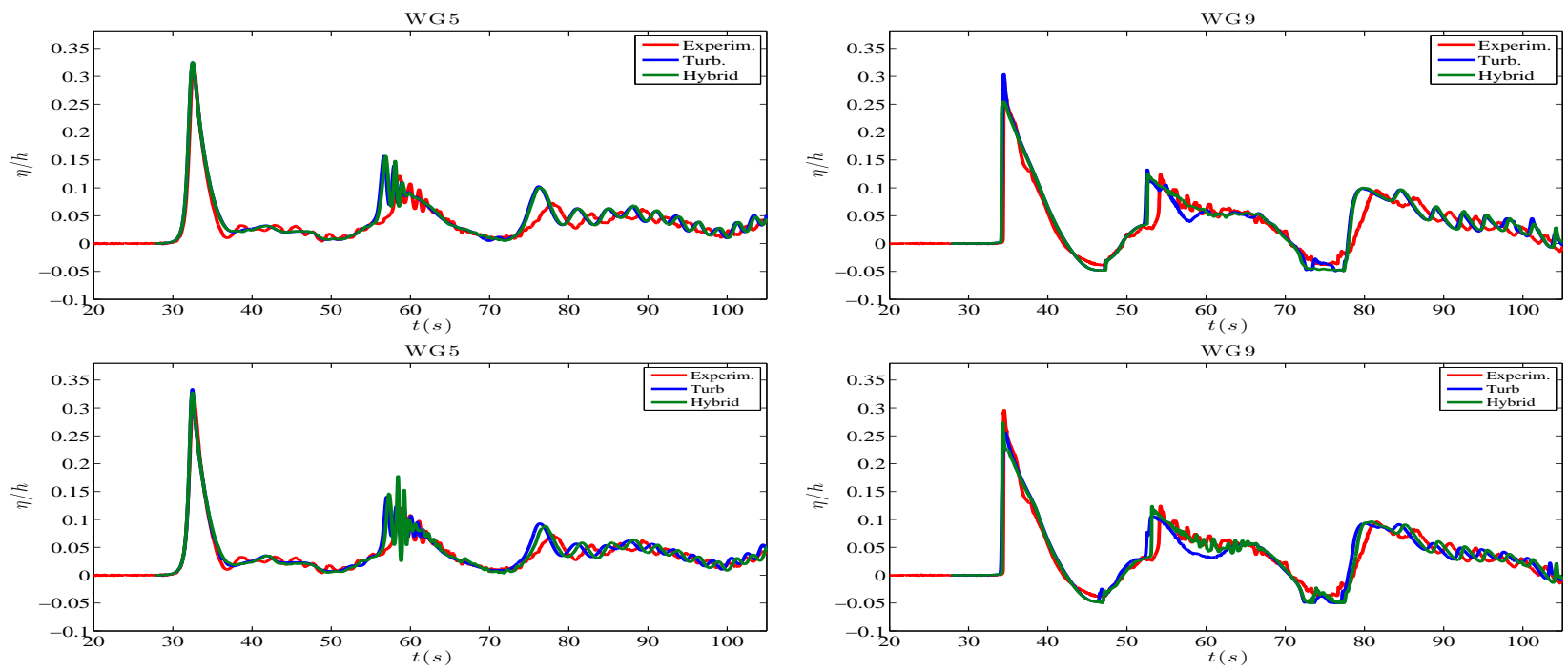

Figure 31: Overtopping of a 2D reef. Free surface time series in wave gauges WG5 (left) and WG9 (right). Top row: GN model. Bottom row: Nwogu model. Blue lines: TKE breaking closure. Green Lines: hybrid wave breaking closure.

ergy preserving approximations in the propagation region), as well as improved models for both the propagation and for the breaking. The models considered at the moment present a dependence on the parameters of the detection criteria, as well as on the coefficients of the TKE equation. Improved models, including the effects of vertical variations of the flow in both the propagation and breaking may be considered in future studies (see e.g. [27, 28,35]). The multi-dimensional case will also have to be studied with attention. In this case more complex effects may come into the picture, related to the interaction with transversal variations of the bathymetry (see e.g. [102]). These effects, and their interaction with the breaking closure will have to be assessed systematically.

\section{Acknowledgements}

Work partially funded by the TANDEM contract, reference ANR-11-RSNR-0023-01 of the French Programme Investissements d'Avenir.

\section{References}

[1] Okey G. Nwogu. Numerical prediction of breaking waves and currents with a Boussinesq model. In Proceedings 25th International Conference on Coastal Engineering, 1996.

[2] A. Demirbilek, Z. Zundel and O. Nwogu. Bouss-2d wave model in the sms: I. graphical interface. coastal and hydraulics laboratory technical note chetni- 69. vicksburg. IMS: U.S. Army Engineer Research and Development Center, 2005.

[3] A. Demirbilek, Z. Zundel and O. Nwogu. Boussinesq modeling of wave propagation and runup over fringing coral reefs, model evaluation report. coastal and hydraulics laboratory technical note chltr0712. vicksburg. IMS: U.S. Army Engineer Research and Development Center, 2007. 

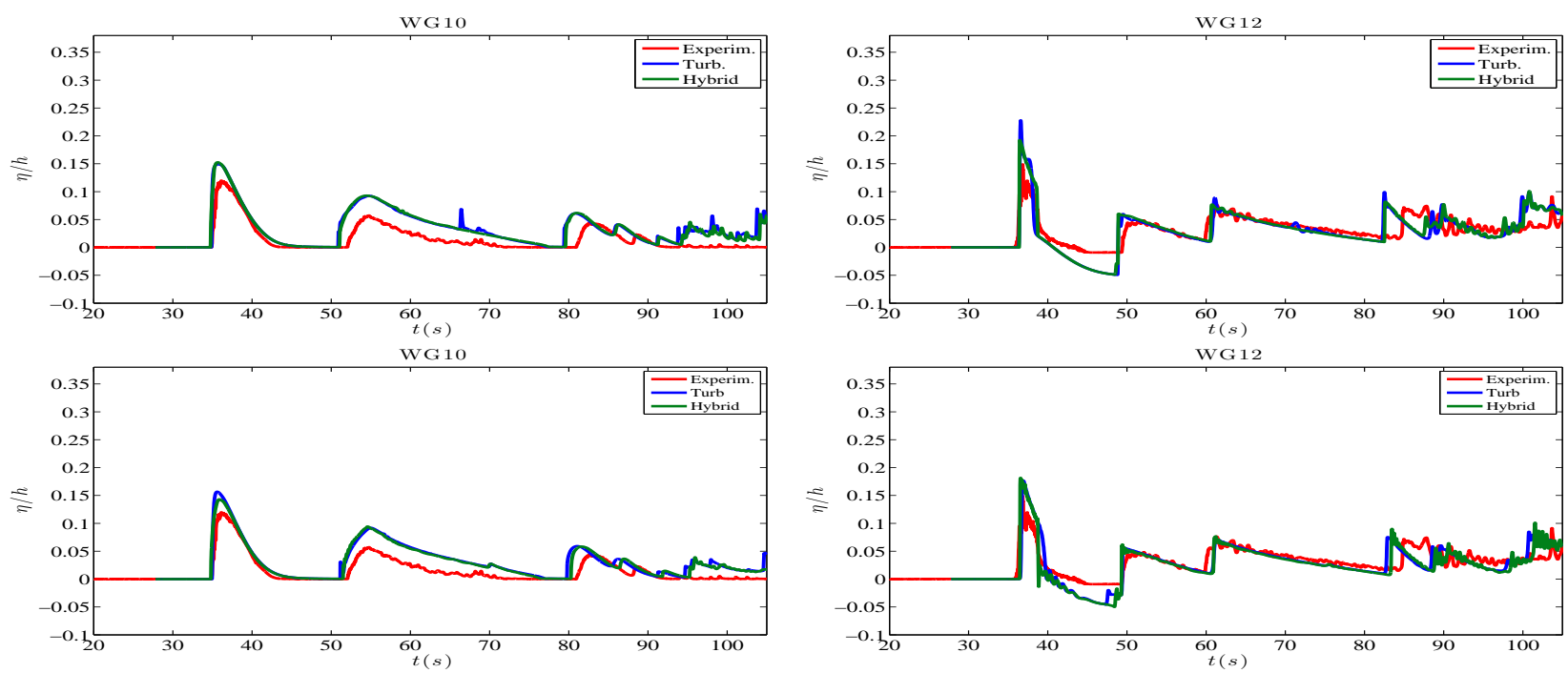

Figure 32: Overtopping of a 2D reef. Free surface time series in wave gauges WG10 (left) and WG12 (right). Top row: GN model. Bottom row: Nwogu model. Blue lines: TKE breaking closure. Green Lines: hybrid wave breaking closure.

[4] Zeki Demirbilek and Okey G Nwogu. Boussinesq modeling of wave propagation and runup over fringing coral reefs, model evaluation report. Technical report, ENGINEER RESEARCH AND DEVELOPMENT CENTER VICKSBURG MS COASTAL AND HYDRAULICS LAB, 2007.

[5] G. Wei and J. T. Kirby. A time-dependent numerical code for extended Boussinesq equations. Journal of Waterway, Port, Coastal, and Ocsean Engineering, 120:251-261, 1995.

[6] F. Shi, J. T. Kirby, J. C. Harris, J. D. Geiman, and S. T. Grilli. A high-order adaptive time-stepping tvd solver for boussinesq modeling of breaking waves and coastal inundation. Ocean Modelling, 43-44:36-51, 2012.

[7] P. Lynett and P.L.-F. Liu. A numerical study of submarine-landslide-generated waves and run-up. Proceedings of the Royal Society of London A: Mathematical, Physical and Engineering Sciences, 458(2028):2885-2910, 2002.

[8] K.I. Sitanggang and P. Lynett. Parallel computation of a highly nonlinear boussinesq equation model through domain decomposition. International Journal for Numerical Methods in Fluids, 49(1):5774, 2005.

[9] V. Roeber and K.F. Cheung. Boussinesq-type model for energetic breaking waves in fringing reef environment. Coast. Eng., 70:1-20, 2012.

[10] DHI Developers Group. MIKE21 Wave Modelling. MIKE 21 Boussinesq Wave Module. https://www.mikepoweredbydhi.com/download/product-documentation.

[11] M. Kazolea, A. I. Delis, I. A Nikolos, and C. E. Synolakis. An unstructured finite volume numerical scheme for extended 2D Boussinesq-type equations. Coast. Eng., 69:42-66, 2012. 
[12] M. Kazolea, A. I. Delis, and C. E. Synolakis. Numerical treatment of wave breaking on unstructured finite volume approximations for extended Boussinesq-type equations. J.Comput.Phys., 271:281$305,2014$.

[13] M. Brocchini. A reasoned overview on Boussinesq-type models: the interplay between physics, mathematics and numerics. Proc. R. Soc. A, 469 (20130496):dx.doi.org/10.1098/rspa.2013.0496, 2013.

[14] J.T. Kirby. Boussinesq models and their application to coastal processes across a wide range of scales. Journal of Waterway, Port, Coastal and Ocean Engineering, 142(6), 2016.

[15] D. Lannes. The water waves Problem, Mathematical Analysis and Asymptotics. American Mathematical Society, Mathematical surveys and monographs, 2013.

[16] J. A. Zelt. The run-up of nonbreaking and breaking solitary waves. Coastal Eng., 15:205-246, 1991.

[17] A. B. Kennedy, J .T. Chen, Q. Kirby, and R. A. Dalrymple. Boussinesq modeling of wave transformation, breaking and runup. Part I: 1D. J. Waterw., Port, Coast., Ocean Engrg., 126:39-47, 2000.

[18] V. Roeber, K. F. Cheung, and M. H. Kobayashi. Shock-capturing Boussinesq-type model for nearshore wave processes. Coast. Eng., 57:407-423, 2010.

[19] G. Wei and J. T. Kirby. A coastal processes model based on time-domain Boussinesq equations. Research report no. CACR-96-01, Center for applied and coastal research, 1996.

[20] Th. V. Karambas and C. Koutitas. A breaking wave propagation model based on the Boussinesq equations. Coastal Engineering, 18:1-19, 1992.

[21] P. J. Lynett. Nearshore Wave Modeling with High-Order Boussinesq-Type Equations. Journal of Waterway, Port, Coastal, and Ocean Engineering, 132:348-357, 2006.

[22] P. J. Lynett, T. R. Wu, and P. L. F. Liu. Modeling wave runup with depth integrated equations. Coastal Eng., 46:98-107, 2002.

[23] R. Cienfuegos, E. Barthélemy, and P. Bonneton. Wave-breaking model for Boussinesq-type equations including roller effects in the mass conservation equation. J. Waterw., Port, Coast., Ocean Engrg., 136:10-26, 2010.

[24] I. Svendsen. Mass flux and undertow in a surf zone. Coastal. Eng., 8:347-365, 1984.

[25] O. R. Sørensen, H. A. Schäffer, and P. A. Madsen. Surf zone dynamics simulated by a Boussinesq type model: Part III. Wave-induced horizontal nearshore circulations. Coastal. Eng., 33:155-176, 1998.

[26] P.A. Madsen, O. R. Sørensen, and H. A. Schäffer. Surf zone dynamics simulated by a Boussinesqtype model: Part II. Surf beat and swash oscillations for wave groups and irregular waves. Coast. Eng., 32:289-319, 1997b.

[27] R. Briganti, R. E. Musumeci, G. Belloti, M Brocchini, and E. Foti. Boussinesq modeling of breaking waves: Description of turbulence. J. Geophys. Res., 109, 2004. 
[28] A. Viviano, R. E. Musumeci, and E. Foti. A nonlinear rotational, quasi-2dh, numerical model for spilling wave propagation. Applied Mathematical Modelling, 39:1099-1118, 2015.

[29] A. Castro and D. Lannes. Fully nonlinear long-wave models in the presence of vorticity. J. Fluid Mech., 759:642-675, 2014.

[30] D. Lannes and F. Marche. Nonlinear wave-current interactions in shallow water. Studies in applied mathematics, 136:382-423, 2016.

[31] M. Kazolea. Mathematical and computational modeling for the generation and propagation of waves in marine and coastal environments. PhD thesis, Technical University of Crete, 2013.

[32] A. Elnaggar, Z. Watanabe. Nonlinear Wave Dynamics in Surf and Swash Zones. In Proceedings 27h International Conference on Coastal Engineering, 2000.

[33] Y. Zhang, Kennedy A. B., A. S. Donahue, J. J. Westerink, and N. Panda. Rotational surf zone modeling for $o\left(\mu^{4}\right)$ Boussinesq-Green-Naghdi systems. Ocean Modelling, 79:43-53, 2014.

[34] G.L. Richard and S.L. Gavrilyuk. Modelling turbulence generation in solitary waves on shear shallow water flows. Journal of Fluid Mechanics, 773:49?74, 2015.

[35] S. L. Gavrilyuk, V. Yu. Liapidevskii, and A. A. Chesnokov. Spilling breakers in shallow water: applications to favre waves and to the shoaling and breaking of solitary waves. Journal of Fluid Mechanics, 808:441-468, 2016.

[36] M. Tonelli and M. Petti. Hybrid finite-volume finite-difference scheme for 2DH improved Boussinesq equations. Coast. Eng., 56:609-620, 2009.

[37] A.G. Filippini, M. Kazolea, and M. Ricchiuto. A flexible genuinely nonlinear approach for nonlinear wave propagation, breaking and runup. J. Comp. Phys., 310:381-417, 2016.

[38] D. Lannes and F. Marche. A new class of fully nonlinear and weakly dispersive Green-Nagdi models for efficient 2d simulations. J. Comp. Phys., 282:238-268, 2015.

[39] J. Kim, J. K. Pederson, F. Lovholt, and LeVeque R. J. A Boussinesq type extension of the GeoClaw model - a study of wave breaking phenomena applying dispersive long wave models. Coastal Eng., 122:75-86, 2017.

[40] A. G. L. Borthwick, M. Ford, B. P. Weston, P. H. Taylor, and P. K. Stansby. Solitary wave transformation, breaking and run-up at a beach. Maritime Engineering, 159:97-105, 2006.

[41] P. Bacigaluppi, M. Ricchiuto, and P. Bonneton. A 1d stabilized finite element model for nonhydrostatic wave breaking and run-up. In J. Fuhrmann, M. Ohlberger, and C. Rohde, editors, Finite Volumes for Complex Applications VII, volume 77 of Springer Proceedings in Mathematics and Statistics. Springer, 2014.

[42] A. Duran and F. Marche. Discontinuous-Galerkin discretization of a new class of Green-Naghdi equations. Communications in Computational Physics, Global Science Press, page 130, 2014.

[43] A. Duran. Tsunami propagation: Numerical issues 1. well balancedness, positivity preservation, high order of accuracy. In TANDEM and DEFIS LITTORAL Tsunami school, Bordeaux, France, 2016. 
[44] F. Marche. Personal communication.

[45] J.T. Kirby et al. B'waves 2014 conference: open discussion session.

[46] Gallerano F., G. Cannata, and Villani M. An integral contravariant formulation of the fully non-linear boussinesq equations. Coast. Eng., 83:119-136, 2014.

[47] C. E. Synolakis. The run up of solitary waves. J. Fluid Mech., 185:532-545, 1987.

[48] Gallerano F., G. Cannata, and Lasaponra F. A new numerical model for simulations of wave transformation, breaking and long-shore currents in complex coastal regions. Int. J. Numer. Methods Fluids, 80:571-613, 2016.

[49] O. Nwogu. An alternative form of the Boussinesq equations for nearshore wave propagation. Journal of Waterway, Port, Coastal, and Ocean Engineering, 119:618-638, 1994.

[50] A.E. Green and P. M. Naghdi. A derivation of equations for wave propagation in water of variable depth. J. Fluid Mech., 78:237-246, 1976.

[51] B. Alvarez-Samaniego and D. Lannes. A Nash-Moser theorem for singular evolution equations. Application to the Serre and Green-Naghdi equations. Indiana University MAthematics Journal, 57(1), 2008.

[52] P. Bonneton, F. Chazel, D. Lannes, F. Marche, and M. Tissier. A splitting approach for the fully nonlinear and weakly dispersive green-naghdi model. Journal of Computational Physics, 230, 2011.

[53] N.P. Waterson and H. Deconinck. Design principles for bounded higher-order convection schemes, a unified approach. J.Comput.Phys., 224(1):182 - 207, 2007.

[54] M. J. Kermani, A. G. Geber, and J. M. Stockie. Thermodynamically based moisture prediction using roes scheme. In The 4th Conference of Iranian AeroSpace Society, 2003. Amir Kabir University of Technology, Tehran, Iran, January 2729.

[55] P. L. Roe. Approximate Riemann solvers, parameter vectors, and difference schemes. J. Comp. Phys., 43:357-372, 1981.

[56] A. Harten. High resolution schemes for hyperbolic conservation laws. J. Comp. Phys., 49:1, 1983.

[57] A. Harten and P. Hyman. Self-adjusting grid methods for one-dimensional hyperbolic conservation laws. J. Comp. Phys., 50:235, 1983.

[58] A. Bermudez and M.E. Vazquez. Upwind methods for hyperbolic conservation laws with source terms. Computers \& Fluids, 23(8):1049 - 1071, 1994.

[59] M. J. Castro, A. M. Ferreiro, J. A. García-Rodriguez, J. M. González-Vida, J. Macías, C. Parés, and M. E. Vázquez-Cendón. The numerical treatment of wet/dry fronts in shallow flows: Application to one-layer and two-layer systems. Mathematical and Computer Modelling, 42:419-439, 2005.

[60] M. Kazolea and A. I. Delis. A well-balanced shock-capturing hybrid finite volume-finite difference numerical scheme for extended 1D boussinesq models. Applied Numerical Mathematics, 67:167186, 2013. 
[61] A. Duran, F. Marche, and Q. Liang. On the well-balanced numerical discretization of shallow water equations on unstrucured meshes. J. Comput. Phys, 235:565-586, 2013.

[62] L. Arpaia and M. Ricchiuto. radaptation for shallow water flows: conservation, well balancedness, efficiency. Computers \& Fluids, 2017. https://doi.org/10.1016/j.compfluid.2017.10.026.

[63] M. Filippini, A.G. Kazolea and M. Ricchiuto. A flexible genuinely nonlinear approach for nonlinear wave propagation, breaking and runup on unstructured grids. In 27th International Offshore and Polar Engineering Conference (ISOPE), June 2017.

[64] M. Ricchiuto and A. Bollermann. Stabilized residual distribution for shallow water simulations. J.Comput.Phys, 228:1071-1115, 2009.

[65] M. Tissier, P. Bonneton, F. Marche, F. Chazel, and D. Lannes. A new approach to handle wave breaking in fully non-linear Boussinesq models. Coastal Engineering, 67:54-66, 2012.

[66] A. Harten. On the symmetric form of systems of conservation laws with entropy. J. Comput. Phys., 49:151-164, 1983.

[67] E. Tadmor. Skew-selfadjoint form for systems of conservation laws. J. Math. Anal. Appl., 103:428$442,1984$.

[68] E. Tadmor. Entropy functions for symmetric systems of conservation laws. J. Math. Anal. Appl., 122:355-359, 1987.

[69] E. Tadmor and W. Zhong. Energy-Preserving and Stable Approximations for the Two-Dimensional Shallow Water Equations, pages 67-94. Springer Berlin Heidelberg, Berlin, Heidelberg, 2008.

[70] U.S. Fjordholm, S. Mishra, and E. Tadmor. Well-balanced and energy stable schemes for the shallow water equations with discontinuous topography. Journal of Computational Physics, 230(14):5587 5609, 2011.

[71] N. Wintermeyer, A.R. Winters, G.J. Gassner, and D.A. Kopriva. An entropy stable nodal discontinuous galerkin method for the two dimensional shallow water equations on unstructured curvilinear meshes with discontinuous bathymetry. Journal of Computational Physics, 340:200 - 242, 2017.

[72] E. Tadmor. The numerical viscosity of entropy stable schemes for systems of conservation laws $i$. Math.Comp., 49:91-103, 1987.

[73] L.L.J. Pratt and J.A. Whitehead. Rorating Hydraulics, volume 36 of Atmospheric and Oceanographic Sciences Library. Springer-Verlag, New-York, 2007.

[74] P. Bonneton. Modelling of periodic wave transformation in the inner surf zone. Ocean Engineering, 34(10):1459 - 1471, 2007.

[75] M. Tonelli and M. Petti. Finite volume scheme for the solution of 2D extended Boussinesq equations in the surf zone. Ocean. Eng., 37:567-582, 2010.

[76] M. Brocchini and D.H. Peregrine. The dynamics of strong turbulence at free surfaces. part 2. freesurface boundary conditions. J. Fluid Mech., 449:255-290, 2001. 
[77] Brocchini M. Free surface boundary conditions at a bubbly/weakly splashing air-water interface. Physics of fluids, 14:1834-1840, 2002.

[78] S.K. Misra, M. Brocchini, and J.T. Kirby. Turbulent interfacial boundary conditions for spilling breakers. In Proceedings of the Coastal Engineering Conference, 2007.

[79] G. Wei, J.T. Kirby, S. T. Grilli, and R. Subramanya. A fully nonlinear Boussinesq model for surface waves. Part 1. Highly nonlinear unsteady waves. Journal of Fluid Mechanics, 294:71-92, 1995.

[80] S. B. Pope. Turbulent Flows. Cambridge University Press, Cambridge, 2003.

[81] F. R. Menter and Y. Egorov. The scale-adaptive simulation method for unsteady turbulent flow predictions. part 1: Theory and model description. Flow, Turbulence and Combustion, 85(1):113$138,2010$.

[82] K.S. Abdol-Hamid. Assessments of - Turbulence Model Based on Menter's Modification to Rotta's Two-Equation Model. International Journal of Aerospace Engineering, 2015, 2015. Article ID 987682, doi:10.1155/2015/987682.

[83] R. J. LeVeque. Finite Volume Methods for Hyperbolic Problems. Cambridge University Press, 2002.

[84] A. Ali and H. Kalisch. Mechanical balance laws for boussinesq models of surface water waves. Journal of Nonlinear Science, 22(3):371-398, Jun 2012.

[85] T.J. Barth. Numerical methods for gasdynamic systems on unstructured meshes. In Kröner, Ohlberger, and Rohde, editors, An Introduction to Recent Developments in Theory and Numerics for Conservation Laws, volume 5 of Lecture Notes in Computational Science and Engineering, pages 195-285. Springer-Verlag, Heidelberg, 1998.

[86] Q. Wang, Z. Zhang, X. Zhang, and Q. Zhu. Energy-preserving finite volume element method for the improved boussinesq equation. Journal of Computational Physics, 270:58 - 69, 2014.

[87] Chaolong Jiang, Jianqiang Sun, Xunfeng He, and Lanlan Zhou. High order energy-preserving method of the "good" boussinesq equation. Numerical Mathematics: Theory, Methods and Applications, 9(1):111?122, 2016.

[88] Jin-Liang Yan, Qian Zhang, Zhi-Yue Zhang, and Dong Liang. A new high-order energy-preserving scheme for the modified korteweg-de vries equation. Numerical Algorithms, 74(3):659-674, Mar 2017.

[89] J. Yan and L. Zheng. New energy-preserving finite volume element scheme for the Korteweg de Vries equation. International Journal of Applied Mathematics, 47(2):223-232, 2017.

[90] G. Wei, J. T. Kirby, and A. Sinha. Generation of waves in Boussinesq models using a source function approach. Coastal Eng., 36:271, 1999.

[91] S. Beji and J. A. Battjes. Experimental investigations of wave propagation over a bar. Coastal Eng., 19:151, 1993.

[92] G.Th. Klonaris, C.D. Memos, and Th.V. Karambas. A boussinesq-type model including wavebreaking terms in both continuity and momentum equations. Ocean Engineering, 57:128 - 140, 2013. 
[93] A. Fillipini, S. Bellec, M. Colin, and M. Ricchiuto. On the nonlinear behaviour of Boussinesq type models: Amplitude-velocity vs amplitude flux forms. Coastal Eng., 99:109-123, 2015.

[94] S.T. Grilli, R. Subramanya, I.A. Svendsen, and J. Veeramony. Shoaling of solitary waves on plane beaches. Journal of Waterway, Port, Coastal and Ocean Engineering, 1994.

[95] D. Arnaud and F. Marche. A discontinuous Galerkin method for a new class of Green-Naghdi equations on simplicial unstructured meshes. Applied Mathematical Modelling, In press.

[96] R. Cienfuegos, E. Barthélemy, and P. Bonneton. A fourth-order compact finite volume scheme for fully nonlinear and weakly dispersive Boussinesq-type equations. Part II: Boundary conditions and validation. Int. J. Numer. Methods Fluids, 53:1423-1455, 2007.

[97] M. Tissier, P. Bonneton, F. Marche, F. Chazel, and D. Lannes. Nearshore Dynamics of Tsunami-like Undular Bores using a Fully Nonlinear Boussinesq Model. Journal of Coastal Research, Special Issue, 64, 2011.

[98] J. B. Hansen and I. A. Svendsen. Regular waves in shoalling water: experimental data. Technical Report, ISVA Series Paper 21, 1979.

[99] V. Roeber. Boussinesq-type model for nearshore wave processes in fringing reef environment. $\mathrm{PhD}$ thesis, University of Hawaii, 2010.

[100] M. Tonelli and M. Petti. Simulation of wave breaking over complex bathymerties by a Boussinesq model. J. Hydraulic Res, 49:473-486, 2011.

[101] M. Kazolea, A.G Filippini, M. Ricchiuto, A. Stéphane, M. M. Medina, D. Morichon, C. Journeau, R. Marcer, K. Pons, L. R. Sylvestre, R. Pedreros, and Rousseau M. Wave propagation breaking, and overtoping on a 2D reef : a comparative evaluation of numerical codes for tsunami modelling. Research Report RR-9005, January 2017.

[102] D.I. Ketcheson and M. Quezada de Luna. Diffractons: Solitary waves created by diffraction in periodic media. SIAM Multiscale Model. Simul., 13(1):440-458, 2015. 